\title{
Integral imaging using phase-only LCoS spatial light modulators as Fresnel lenslet arrays
}

\author{
Ali Özgür Yöntem* and Levent Onural \\ Department of Electrical and Electronics Engineering, Bilkent University, Ankara TR-06800, Turkey \\ *Corresponding author: aozgur@ee.bilkent.edu.tr
}

Received June 10, 2011; revised September 13, 2011; accepted September 15, 2011; posted September 16, 2011 (Doc. ID 148975); published October 27, 2011

\begin{abstract}
We present a digital integral imaging system. A Fresnel lenslet array pattern is written on a phase-only LCoS spatial light modulator device (SLM) to replace the regular analog lenslet array in a conventional integral imaging system. We theoretically analyze the capture part of the proposed system based on Fresnel wave propagation formulation. Because of pixelation and quantization of the lenslet array pattern, higher diffraction orders and multiple focal points emerge. Because of the multiple focal planes introduced by the discrete lenslets, multiple image planes are observed. The use of discrete lenslet arrays also causes some other artifacts on the recorded elemental images. The results reduce to those available in the literature when the effects introduced by the discrete nature of the lenslets are omitted. We performed simulations of the capture part. It is possible to obtain the elemental images with an acceptable visual quality. We also constructed an optical integral imaging system with both capture and display parts using the proposed discrete Fresnel lenslet array written on a SLM. Optical results when self-luminous objects, such as an LED array, are used indicate that the proposed system yields satisfactory results. (C) 2011 Optical Society of America
\end{abstract}

OCIS codes: $\quad 110.0110,110.4190,110.6880$.

\section{INTRODUCTION}

Long after Lippmann had proposed integral imaging [1] , it became a popular research topic [2] and now it is used as a 3D autostereoscopic capture and display method. As CCD arrays and LCDs emerged, digital implementations of Lippmann's original work were also reported [3]. Chemical photographic capture and display processes are now almost entirely replaced by these digital recording and display devices. Today, the resolution and size of these digital devices are sufficiently high for experimental capture and display of small-sized 3D objects/scenes. Even if the resolution of these devices is not yet comparable to that of chemical photographic emulsions, the perceived $3 \mathrm{D}$ object quality is quite good. Such devices are getting more and more popular due to well-known advantages, such as flexibility, and also due to the easy reproducibility, processing, storage, and transmission of the data written on these devices. Current research focus in integral imaging is mainly on quality improvements of perceived 3D objects/scenes by changing the physical properties of the lenslet arrays [4,5]. One of the problems is the small viewing angle. A structure composed of a curved screen and a curved lenslet array is proposed [6] to overcome this problem. Since it is difficult to produce such lenses, placing a large aperture lens, which simulates a curved array, in front of a planar array of lenses was proposed [7]. Another issue is the limited depth of field. This problem is due to the physical properties of the lenslets in the array. It is shown that by using amplitude masks the depth of focus of the system can be increased by trading-off lateral resolution and light throughput [ㄴ,, 8$]$. It is also possible to use phase masks on the lenslets to improve the depth range of the system [5]. Another problem of integral imaging is pseudoscopic 3D object perceived at the display end. The simplest practical solution is to replicate the process once more to obtain an orthoscopic image [2]. However, this makes the system or the process cumbersome. So, digital and optical solutions to this problem are proposed in $[3,9]$, respectively. Even if those issues are fundamentally important to improve the perceived image quality, the generic system did not change much. The key element of the system, lenslet array, is still mostly an analog device. Usually, it is a fixed component with fixed physical parameters. So, if we need to change the physical parameters of the lenslet array, it should be manufactured again. Furthermore, different types of lenslets to overcome some problems like the insufficient depth of field may be needed [8]. It is difficult to manufacture such special lenslet arrays. It would be much easier to program an electronic device that would act as an electronic lenslet array. Fortunately, it is shown that for adaptive optics, programmable lenslet arrays can easily be implemented using LCoS phase-only SLMs and are used in Hartmann-Shack sensors $[10,11]$. Such devices work in the phase-only mode so that Fresnel lenses can be written on them [12-14]. In some early studies, magneto-optic SLMs were used to write binary Fresnel lens patterns [15] and used to modulate light. Moreover, it is shown that it is possible to generate such lenslet arrays [16]. It is also mentioned in [16] that a generated lenslet array is used to image a $3 \mathrm{D}$ object. However, experimental results were not given. In [17], it is presented that electronically synthesized Fresnel lenslet arrays can be encoded on an LCD panel for integral imaging. In that paper, they showed the potential of the idea by applying it to their previous setup, which increases the viewing angle by mechanically moving the lenslet array. In theory, the electronic lenslet array replaces the moving lenslet array. However, because of the physical limitations of the LCD panel, it is reported that such lenslet arrays were not used in the optical experiments of the pickup process. It is also reported that perceived resolution of the $3 \mathrm{D}$ 
reconstruction with the bare eye with the above-mentioned system was very low. They suggested that an SLM with smaller pixel size would give better results.

In this paper, we analyze the effects of using pixelated and quantized lenslet arrays in an integral imaging system. Specifically, we find the analytical results for the output elemental images of the capture stage with a self-luminous 3D point cloud input. We carried the analysis as if the source points are coherent sources. The pixelated and quantized lenslet arrays introduce some artifacts. Some of these artifacts are multiple focal lengths due to quantization, and higher-order diffractions due to the pixelated structure of the lenslets. There is also inherent apodization due to the finite pixel size [18]. We carry out the analysis by taking into consideration these features of pixelated and quantized lenslet arrays, and show that when these effects are ignored, the results simplify to the previous results given in the literature $[4,8]$. We run simulations to confirm the theoretical results and they are in good agreement. Furthermore, we show that we can construct a versatile integral imaging system by using a programmable lenslet array, which is formed by writing an array of Fresnel lenslet phase profiles on a high definition $(1920 \times 1080)$ reflective phase-only LCoS SLM (Holoeye HEO 1080P); this replaces the conventional lenslet array. Furthermore, we present theoretical background for the system. An integral imaging system, which implements the display as a reflective imaging scheme, is proposed in the literature [19]. In that system, a concave mirror array replaces the lenslet array and the image is formed by the help of a half mirror. The elemental images on a 2D display are integrated at the reconstruction distance that is not on the same optical axis with the elemental images. The half mirror folds the optical axis by 90 degrees. The reconstructed 3D object is formed away from the half mirror. In our system we use a similar scheme since our SLM device is also reflective type. Both of the capture and display parts of our system work with half mirrors. The elemental images and the reconstructed images of the capture and the display systems are formed away from the half mirrors. However, we use a $2 \mathrm{D}$ lenslet array phase profile that is written on the SLM electronically instead of a concave mirror array. This way we succeeded to implement the entire integral imaging structure as a digital system. We believe that this approach increases the capability and flexibility of the system, significantly. Thus, all subsequent improvements to increase the system quality can be implemented easily by electronically changing the lenslet array structure using digital means. In Section 2, we present the formulation for lenslet array patterns with certain focal lengths and describe the properties of such discrete lenslets. We review the multiple focal points issue due to quantization. We also analyze the capture system from a signal processing perspective and give a brief explanation for the display system. In Subsection 3.A, we present the results of computer simulations and give correspondences to the theoretical results. In Subsection 3.B, we show the proposed systems for both capture and display parts of the integral imaging setup for specific physical parameters and describe the optical setup and present the optical experiment results. Finally we draw conclusions in Section 4 .

\section{ANALYSIS}

The purpose of this section is to form the theoretical background of a conventional integral imaging setup, where an analog lenslet array is replaced by an LCoS SLM, which has an array of Fresnel lenslets written on it. Since the SLM has a discrete nature we need to determine the $2 \mathrm{D}$ discrete array that will be written on the SLM. The $2 \mathrm{D}$ discrete pattern of Fresnel lenslet array written on the SLM is calculated by first sampling a quadratic phase function with certain parameters to represent a single Fresnel lenslet, then, by quantizing the sample values to match the phase levels that the SLM can support, and finally, by replicating as many single lenslets in $2 \mathrm{D}$ as the SLM dimensions can support. Before the analysis of the capture setup, we reviewed the response of a single sampled and quantized lenslet and also an array of such lenslets to a plane wave illumination. For the continuous field propagation analysis, we converted the $2 \mathrm{D}$ discrete pattern to a continuous field by interpolating it with the pixel function related to the SLM. In the capture part analysis of the integral imaging setup, first the impulse response of the system is determined and then a 3D point cloud is imaged and the intensity distribution at the elemental image plane is given using a lenslet array on the SLM. The relation between the parameters in the mathematical models to the physical setup is discussed.

\section{A. Quadratic Discrete Phase Array Patterns}

It is crucial to determine how to generate the lenslet array. Under the Fresnel approximation, the phase pattern of a thin lens is a quadratic phase function. For the simplest case let us consider a sampled (discrete) thin lens phase pattern written on an SLM device. For a lens with a focal length $f$, the continuous quadratic phase function is

$$
h_{-\gamma}(\mathbf{x})=\exp \left(-j \gamma \mathbf{x}^{T} \mathbf{x}\right),
$$

where $\mathbf{x}=[x y]^{T}, x, y \in \mathbf{R}$, and $\gamma$ is a parameter that we will explain later. By substituting $\mathbf{x}$ by $\mathbf{V n}$ we get the 2D complex discrete lens quadratic phase pattern

$$
h[\mathbf{n}]=\exp \left(-j \gamma \mathbf{n}^{T} \mathbf{V}^{T} \mathbf{V n}\right),
$$

where $\mathbf{V}$ is the $2 \mathrm{D}$ sampling matrix. For simplicity we chose a regular rectangular sampling matrix $V=\left[\begin{array}{cc}X & 0 \\ 0 & X\end{array}\right]$, where $X$ is the sampling period (pixel period of the SLM), and $\mathbf{n}=\left[n_{1} n_{2}\right]^{T}$, where $n_{1}, n_{2}$ are integers. The sampling of the quadratic phase function will cause a very specific type of aliasing and naturally generates an array of Fresnel lens patterns [20]. In [16], lenslet arrays are generated using this method. However, each lenslet in the generated array may have different phase variation relative to its neighbor lenslet due to the parameters of the designed array [16]. In [20], it is shown that sampled quadratic phase patterns may have certain periodicity properties with such phase variations on them. By choosing the parameters properly, one can obtain a periodic phase pattern with no phase variations on them so each lenslet will be exactly the same as its neighbors. Instead of this approach, we designed a single lenslet pattern and then replicated this pattern one after another to cover the entire SLM surface. Therefore, there is no phase variation between the lenslets. Even though this method may cause 
phase jumps at the borders of the lenslets, and, thus, some unwanted effects, such effects are negligible especially for larger array sizes. Furthermore, generation of lenslets with special phase patterns [5] is easier to obtain using this method. Also, such an approach makes it easier to fit a certain array configuration over the finite size SLM. To determine the number of lenslets given the SLM size we need the discrete array size of a single lenslet pattern. For this reason, we need to relate the parameter $\gamma$ to physical parameters as $\gamma=\frac{\pi}{\lambda f}$, where $\lambda$ is the wavelength. Focal length should be chosen as $f=N \frac{X^{2}}{\lambda}$ to cover the entire normalized local frequency range $[-\pi, \pi)$, in radians, for the discrete signal, where $N$ is the number of pixels along one dimension of the finite $2 \mathrm{D}$ discrete array, which represents a single lenslet. So, when we limit $n_{1}, n_{2}$ in Eq. (2) to be in the interval $\left[-\frac{N}{2}, \frac{N}{2}-1\right]$, we will obtain a single lenslet. If the SLM has $N_{1} \times N_{2}$ pixels, then the number of the lenslets will be $\frac{N_{1}}{N} \times \frac{N_{2}}{N}$, where $\frac{N_{1}}{N}$ and $\frac{N_{2}}{N}$ are integers.

For a setup that requires longer focal length lenslets, we can still use the same SLM with the same lenslet array size, by keeping the lenslet size $N \times N$ the same; thus, the Fresnel pattern becomes cropped. Therefore, we will not be able to cover the full local frequency range of $[-\pi, \pi)$ for longer focal length lenslets in this case. This will introduce blurriness since the light that would be coming from higher angles does not exist as a consequence of the cropped lens pattern, and thus, will not be accumulated at the focal point. So, there is a tradeoff between longer focal lengths and focused point sharpness if the size of the lenslet is kept fixed. One can easily calculate the range of instantaneous frequencies, which the lenslet can accommodate with such a larger focal length, of the sampled quadratic phase function. In our experiments, we used several lenslet patterns with focal lengths equal to $N \frac{X^{2}}{\lambda}$ to cover the full local frequency range. In each such pattern, we determined the value of $N$, which specifies $f$ since $\lambda$ and $X$ are fixed, and each lenslet is generated according to these parameters. Our device has $1920 \times 1080$ (HDTV) pixels with a $8 \mu \mathrm{m}$ pixel period in each direction. We were able to implement $3 \times 5,6 \times 10$, and $12 \times 20$ arrays of lenslets, which have $360 \times 360,180 \times 180$, and $90 \times 90$ pixels with $43.4,21.65$, and $10.825 \mathrm{~mm}$ focal lengths, respectively, for the same wavelength of $532 \mathrm{~nm}$. There are some unused pixels on the left and right side of the SLM with the given size and array configurations; we evenly split this excess area to both ends. We were also able to generate shorter focal length lenslet arrays. However, the resultant imaging quality in the optical experiments with these lenslets was low. This is because the higherorder effects (multiple focal points and higher diffraction orders) are dominant; thus, it does not behave as a good quality lens anymore.

\section{B. Multiple Focal Points}

The SLM acts as a diffractive optical element. The pixelated structure of the SLM causes higher diffraction orders; this is a well-known effect $[20,18]$. However, this is not the only effect that we observe when a discrete quadratic phase function is written on the SLM. For writing any pattern onto the SLM we also need to quantize the sampled pattern. The quantization is a nonlinear process and its consequences are investigated in [21] for the quadratic phase function. Suppose that we have a sampled and quantized Fresnel lens pattern that we want to write on an SLM, which has exactly the same amount of pixels as the number of samples of the finite-size pattern. The Fresnel pattern is like a discrete hologram of a point source at a distance $f$. We will call this distance the fundamental focal point. This distance from the lens is also referred as the reference focal [22] and it is also called the critical distance [15]. Between the lenslet and the fundamental focal point, we have to consider the effect of both sampling and quantization together. Quantization will cause multiple focal points over the $z$ axis. When such a discrete and quantized Fresnel pattern is illuminated by a plane wave and the modulated light is allowed to propagate along the $z$ axis the light will be concentrated on bright spots on these focal points [21]. So, quantization causes multiple focal planes while sampling causes higher diffraction orders.

Considering the case of an infinite array of such lenslets, the phase angle of the complex pattern, which is the sampled and quantized Fresnel lenslet array pattern as calculated in the previous section, is written on a hypothetical infinite-size phase-only SLM. When the SLM is illuminated by a plane wave and the modulated light is allowed to propagate further away from the fundamental focal length on the $z$ axis, the periodic array of focused points will be repeated at certain distances. This phenomenon is known as the Talbot effect [23]. When an infinite array of discrete lenslets, each with a size $N \times N$, is written on an infinite-size SLM, so-called self images of the lenslet array will periodically occur at multiples of Talbot distance given as $z_{T}=m \frac{(N X)^{2}}{\lambda}=m N f$ on the $z$ axis, where $N X$ is the distance between two lenslets (lenslet period), and $m$ is an even integer [23]. Since the input pattern will be repeated at multiples of $z_{T}$, the fundamental focal point will also be repeated at multiples of $z_{T}$. Furthermore, between each Talbot distance at $m z_{T}$ and $(m+1) z_{T}$, there exist other spots at fractions of the Talbot distance. In real life, the Talbot effect may or may not be visible physically depending on the length $z_{T}$ and also on the lenslet array size, and thus on the SLM size. For a sufficiently large array and short $z_{T}$, it is possible to observe this effect. For example, in our setup, it is possible to observe the periodic focused spots created by a lenslet array pattern with a $24 \times 40$ lenslet array where each lenslet has $45 \times 45$ pixels, $f=5.41 \mathrm{~mm}$, and the first Talbot distance $z_{T}+f=0.492 \mathrm{~m}$.

To relate the physical observations to theoretical analysis, let us consider a hypothetical case: an infinite-size analog mask of periodic lenslets consisting of equally spaced impulsive elements is illuminated by a plane wave. We use the Fresnel transform as if it is a valid diffraction model. In reality, this model is valid only for small angle propagation (the paraxial approximation) and such a restriction will not support impulsive patterns since they imply high frequencies. Even if the diffraction model is not the Fresnel model but the accurate Rayleigh-Sommerfeld model, the free space propagating waves still do not support an impulsive pattern, since the plane wave components that superpose to form an impulse should inevitably have both propagating and evanescent components. Those components with spatial frequencies $\left(\nu_{x}, \nu_{y}\right)$ on the mask, such that $\sqrt{\nu_{x}^{2}+\nu_{y}^{2}}>1 / \lambda$, will not propagate and result in evanescent waves. Those components whose spatial frequencies satisfy $\sqrt{\nu_{x}^{2}+\nu_{y}^{2}} \leq 1 / \lambda$ will propagate. Therefore, impulsive function cannot be reconstructed by propagating waves. However, here we still conduct a 


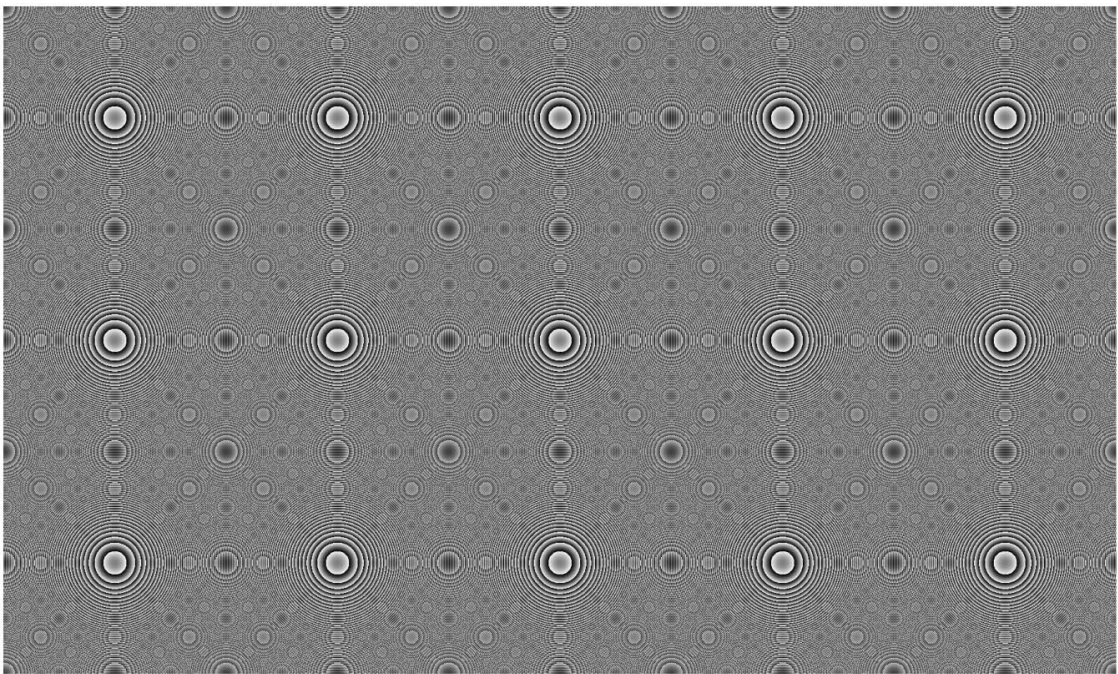

Fig. 1. $3 \times 5$ Lenslet array phase profile on the SLM, each lens has $f=43.3 \mathrm{~mm}$. There are equal number of unused pixels both at left and right edges.

mathematical exercise using impulsive inputs and Fresnel propagation to understand the associated concepts.

We start from a Fourier series expansion [21]. For the sake of simplicity, we look at the $1 \mathrm{D}$ version of the continuous quadratic phase function

$$
h_{-\gamma}(x)=\exp \left(-j \gamma x^{2}\right)
$$

Consider that we make a change of variables $u=x^{2}$. The function $b(u)=\exp (-j \gamma u)$ is a periodic function of $u$. Now, we introduce a pointwise nonlinearity $T(\cdot)$ to get another periodic function $\phi(u)=T[b(u)]$, and then we can make a Fourier series analysis of $\phi(u)$ to find a set of coefficients as

$$
a_{k}=\frac{1}{L} \int_{0}^{L} \phi(u) \exp \left(j \frac{2 \pi k}{L} u\right) \mathrm{d} u
$$

where $L$ is the period $\frac{2 \pi}{\gamma}$. A commonly used nonlinearity is the staircase function, which is investigated in [21]. Following the similar steps in [21], and by substituting back for $u$, the Fourier synthesis can be written as

$$
\begin{aligned}
\psi(x) & =\left.\phi(u)\right|_{u=x^{2}}=\sum_{k} a_{k} \exp \left(-j \frac{2 \pi k}{L} x^{2}\right) \\
& =\sum_{k} a_{k} \exp \left(-j \frac{\pi}{\lambda f_{k}} x^{2}\right) .
\end{aligned}
$$

Equation (5) can be interpreted as many superposed thin lenses with different focal lengths at $f_{k}=\frac{f}{k}$ and different transparencies indicated by the amplitude $a_{k}$. It is possible to design a nonlinear function to achieve a desired allocation of power among the terms of Eq. (5) by choosing $a_{k}$ 's accordingly. Usually, it is desirable to emphasize $a_{1}$ and suppress other terms. For example, it is shown in [21] that by increasing the quantization level it is possible to increase the power contributed to the fundamental focal point (larger $a_{1}$ with respect to other $a_{k}$ 's). However, there still exists power contributed to other focal points since $a_{k}$ 's for $k \neq 1$ are not necessarily zero. Let us concentrate on the imaging properties of a quantized lens with arbitrary $a_{k}$ 's. When we use such a lens to image an object, due to multiple focal lengths, not only the main image, which is formed by the lens with focal length $f$, is present but also there exists images formed by the higher-order lenses with smaller focal lengths. However, these images, which are also smaller in size, have less power as a consequence of distribution of $a_{k}$ 's, as discussed above, and quickly disperse when they propagate and reach the main image plane. We conducted simulations related to this observation and the results are presented in Section 3.A. In our experiments, the quantization is linear with equidistant 256 -levels between 0 and 255 to cover the $[0,2 \pi)$ radians phase interval. Subsequently, we convert $\psi(x)$ to a pixilated form by sampling (multiplying by an impulse train) it first and then convolving the result with a zero-order interpolator (hold), which has a width equal to the sampling period $X$ where we assumed that there are no gaps between the SLM pixels. In case there are gaps, the analysis should be modified by starting as presented in $[\underline{22,18]}$. Therefore, we can write

$$
\psi_{s}(x)=\left[p(x) \psi(x) \sum_{n} \delta(x-n X)\right] * s(x) .
$$

The finite size of a lenslet is represented by the aperture function $p(x)=\operatorname{rect}\left(\frac{x}{N X}+\frac{1}{2}\right) \cdot s(x)=\operatorname{rect}\left(\frac{x}{X}\right)$ is the pixel function, where the rectangular function defined as $\operatorname{rect}(x)=1$ for $x \in$ $[0,1)$ and 0 otherwise. Figure 2 shows the angle of $\psi_{s}(x)$, modulo $2 \pi$.

To create the lenslet array, we replicate the function $p(x) \psi(x)$ by convolving it with an impulse train, which shifts the center of each lenslet in the array such that each lenslet is positioned one next to another:

$$
L A_{s}(x)=\left\{\left[p(x) \psi(x) * \sum_{r} \delta\left(x-r x_{0}\right)\right] \sum_{n} \delta(x-n X)\right\} * s(x),
$$

where $n$ and $r$ are integers. In Eq. (7), we modeled the lenslet array such that there are no gaps between two consecutive lenslets; thus, the lenslet period is equal to the lenslet size. There are $N$ pixels in one direction from the center of one lenslet to next center of the next lenslet. Therefore, the lenslet 


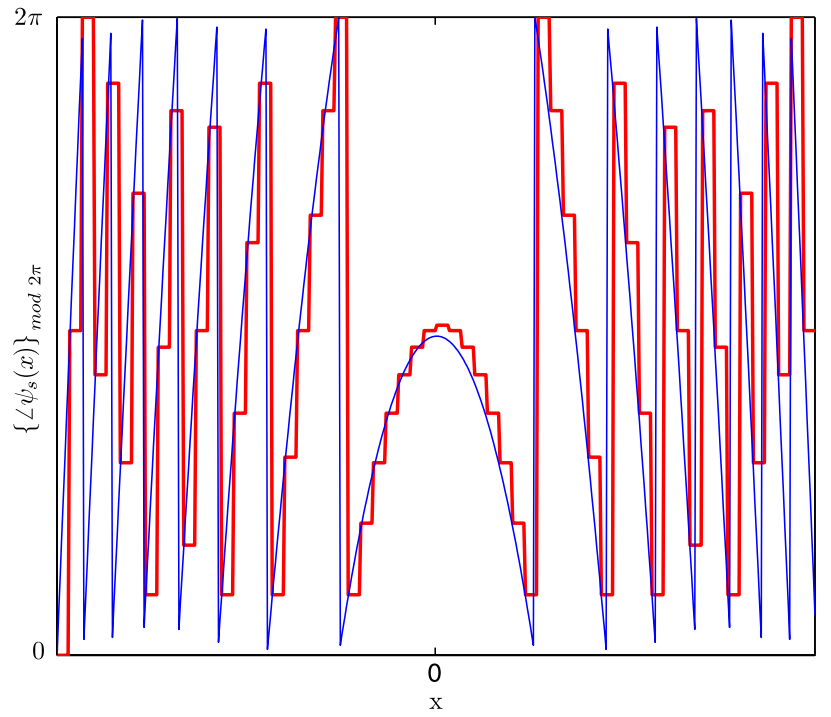

Fig. 2. (Color online) Illustration of a quadratic phase function and its sampled and quantized version. Vertical axis shows the phase, $\bmod 2 \pi$, while the horizontal axis shows the spatial extent of the function.

array period is $x_{0}=N X$. The reason for this choice is the finite SLM size: since we have limited number of pixels, we want to generate as many lenslets as we can without wasting any pixels between the lenslets. Depending on the application, it might be desirable to have gaps between the lenslets. However, the relation between the pixel period and the lenslet period is critical: if this ratio is not an integer, then the focal points from higher diffraction orders, and the multiple focal points due to higher-order lenslets due to nonlinearity will not overlap. They do overlap in our choice as indicated above.

Now assume that the lenslet array is illuminated by a plane wave. Then, the complex field just after the infinite-size SLM will be given by Eq. (7). Therefore, the field that propagates away from the SLM is

$$
q(x)=L A_{s}(x) * h_{\chi}(x)=\int_{\eta} L A_{s}(\eta) h_{\chi}(x-\eta) \mathrm{d} \eta,
$$

where $\chi=\frac{\pi}{\lambda z}$, which indicates the distance along the $z$ direction from the SLM plane. There are two convolutions within the function $L A_{s}(x)$. Using the commutative property of the convolution operation we replace the order of the convolutions $s(x)$ and $h_{\chi}(x)$ in Eq. (8) [18]. So, the above equation can be rewritten as

$$
\begin{aligned}
q(x)= & \left\{\left[p(x) \psi(x) * \sum_{r} \delta\left(x-r x_{0}\right)\right] \sum_{n} \delta(x-n X)\right\} \\
& * h_{\chi}(x) * s(x) .
\end{aligned}
$$

As a consequence, it is easier to observe the focusing properties of a sampled and quantized lenslet array and explain the effect of the rectangular pixels. Carrying out the convolution by $h_{\chi}(x)$ and the function inside the curly brackets, where the evaluation is given in Appendix $\underline{\mathrm{A}}$, we can rewrite Eq. (9) as

$$
\begin{gathered}
q(x)=\sum_{k}\left\{\left[P\left(\frac{x}{\lambda f_{k}}\right) * \frac{x_{0}}{k} \sum_{n} \delta\left(x-\frac{n}{k} x_{0}\right)\right]\right. \\
\left.*\left[\sum_{r} c_{k, r}(x) \delta\left(x-r x_{0}\right)\right]\right\} * s(x),
\end{gathered}
$$

where the parameter $\chi$ is set as $\gamma_{k}$, thus, $z=f_{k}$, (i.e., we have the field at the focal distances) to show the effect of multiple focal planes. $P\left(\frac{x}{\lambda f_{k}}\right)$ is the Fourier transform of the lenslet's pupil function scaled with $\frac{x}{\lambda f_{k}}$; so, for the 1D analysis we have been carrying out, it is given by $\frac{k}{X} \operatorname{sinc}\left(\frac{x}{X} k\right)$ where $\operatorname{sinc}(x)=\frac{\sin (x)}{x}$. It represents the effect on the diffraction due to the limited aperture size of the lenslet. The constants are given as $\frac{\lambda f_{k}}{X}=\frac{N X}{k}=\frac{x_{0}}{k}$ and $c_{k, r}(x)=a_{k} h_{\gamma_{k}}\left(x-r x_{0}\right)$. It is shown in [18] that the output of the diffraction from a single lenslet is the convolution of the Fourier transform of the pupil function and the pixel function, which introduces an inherent apodization [18]. This observation still holds for an array of lenslets shown by Eq. (10), as expected.

In Eq. (10), the impulse train indexed by $n$ is due to the sampling of the lenslet and this introduces multiple diffraction orders. At the fundamental focal plane, that is when $k=1$, we observe that the separation of higher diffraction orders of focused spots of a lenslet is $x_{0}$. In our cases, this period matches the impulse train indexed by $r$, which is present due to the separation of the lenslets. The interesting case occurs due to the effect of quantization since it causes multiple focal points. Note that the impulse train indexed by $n$, when $k \neq 1$, introduces shifts, which are a fraction of $x_{0}$. So, the spots appear on the $x$ axis with $x_{0} / k$ distance away from each other on each focal plane $f / k$. Since all impulse trains are infinite in extent, we see that the focused spots at a certain distance are periodic over the $x$ axis. So, Eq. (10) is a collection of points in threedimensional free space.

Equation (10) can be illustrated by Fig. 3, where the circles along the optical axis of each lenslet specifies the multiple focal points, and along the $x$ axis, at each focal length, periodically positioned spots are present due to higher diffraction orders. For a single lenslet pattern in the lenslet array, any focused spot it yields along the $x$ axis, except the ones that lie on its optic axis, are higher diffraction orders at the focal planes. Thus, each lenslet creates multiple depth focal points, together with higher diffraction orders. In other words, when the generated Fresnel field from the impulsive pattern propagates in the free space, at certain distances, again periodic and impulsive patterns are formed [20].

Now we look at the case of a finite-size SLM. In this case, the locations of the focused spots will not change. However, limited SLM aperture will introduce a low pass filter over the intensities of these spots. Depending on the aperture size, the intensities of the focused spots are modulated by a sinc function $W\left(\frac{x}{\lambda f_{k}}\right)=\frac{K x_{0}}{\lambda f_{k}} \operatorname{sinc}\left(\frac{x}{\lambda f_{k}} K x_{0}\right)=\frac{K k}{X} \operatorname{sinc}\left(\frac{x K k}{X}\right)$, which is due to a rectangular window function $w(x)=\operatorname{rect}\left(\frac{x}{K x_{0}}\right)$, which is the aperture function of the SLM, which contains $K$ discrete lenses along one direction. Such a modulation will diminish the power associated with some of these multiple focused spots and thus reduce their visibility.

Before investigating the impulse response and the imaging properties of quantized and sampled lenslet arrays, we achieved to formulate the focusing properties of such lenslet arrays: quantization causes multiple focal planes while sampling causes higher diffraction orders at each focal plane. This will help us in the next section while formulating the generation of elemental images, since we will follow similar steps. 


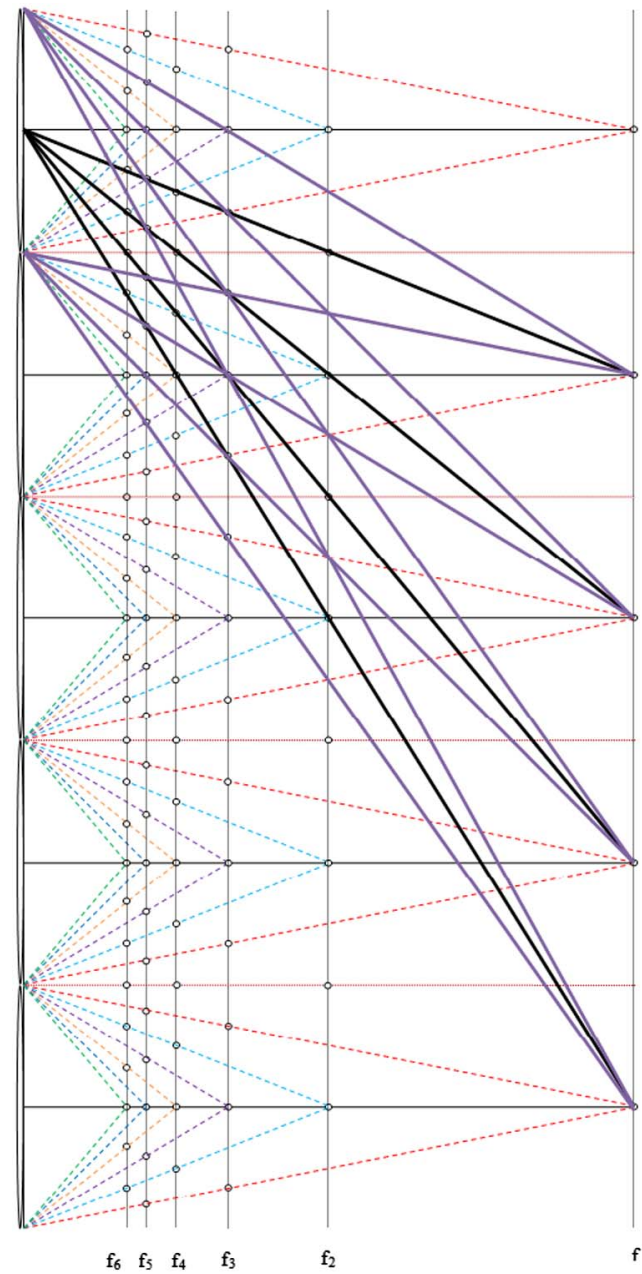

Fig. 3. (Color online) Multiple focal points and higher diffraction orders. The focal points are shown by small circles. Dashed lines show the converging waves toward multiple focal points from a single lenslet. Solid lines show the converging waves toward higher diffraction orders at the fundamental focal plane. (Not all lines are shown in order not to clutter the drawing.)

\section{Capture System Analysis}

This section will provide the analytical results, which give the elemental images of a 3D object defined by a point cloud. In the analysis of the capture system we start from the light that propagates from an object to the SLM plane. The propagated field is then multiplied with the phase profile, which is the lenslet array profile, written on the SLM. The resulting field is then propagated to the recording plane. We will also follow a similar derivation as in Subsection 2.B with the addition of the calculation of the propagation from the object. The object field arriving at the SLM plane replaces the plane wave illumination used in the formulation of the previous section. Again we will use the Fresnel -based wave propagation model. For simplicity, a 3D input object is modeled as a point cloud. Here, we again assume that in the derivations the SLM has impulsive pixels and add the effect of rectangular pixels later. The capture system scheme is shown in Fig. 4 . The distance $d$ is measured from a chosen theoretical reference plane on which the closest point on the object to the lenslet array plane is located. The distance $g$ is measured from the lenslet array plane to the CCD plane. These distances are chosen such that they satisfy

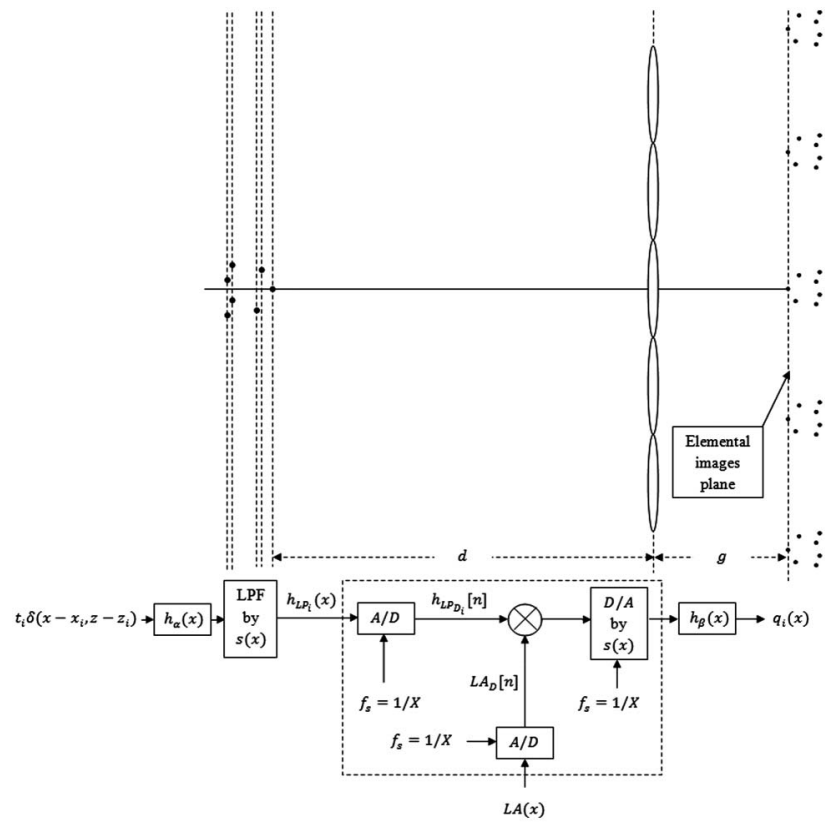

Fig. 4. Capture setup.

the imaging equation $1 / f=1 / d+1 / g$ for a single lenslet with focal length $f$.

The point cloud, which is the input of the system, is defined as

$$
t(x, z)=\sum_{i} t_{i} \delta\left(x-x_{i}, z-z_{i}\right)
$$

where $z_{i}=d+\Delta z_{i}$, which is the depth location of object points. $t_{i}$ 's are the complex valued source amplitudes. Therefore, we assume that the object consists of self-luminous point sources. The complex field just before the lenslet array is given by the sum of the convolutions of the propagation kernel with each point source in the point cloud. On the lenslet array plane, since the physical device consists of pixels, we assume that the light falling onto a pixel is integrated to yield a constant value. Thus, we get the propagated field at the lenslet array plane as

$$
\begin{aligned}
h_{L P_{i}}(x) & =t_{i} h_{\alpha_{i}}(x) * s(x) * \delta\left(x-x_{i}\right) \\
& =t_{i}\left[\int h_{\alpha_{i}}(x-\eta) s(\eta) \mathrm{d} \eta\right] * \delta\left(x-x_{i}\right) \\
& =t_{i}\left[\int h_{\alpha_{i}}(x) \exp \left(-j 2 \alpha_{i} x \eta\right) h_{\alpha_{i}}(\eta) s(\eta) \mathrm{d} \eta\right] * \delta\left(x-x_{i}\right) \\
& =t_{i} h_{\alpha_{i}}(x)\left[\int h_{\alpha_{i}}(\eta) s(\eta) \exp \left(-j 2 \alpha_{i} x \eta\right) \mathrm{d} \eta\right] * \delta\left(x-x_{i}\right) \\
& =\left\{t_{i} h_{\alpha_{i}}(x)\left[H_{\alpha_{i}}\left(\frac{x}{\lambda z_{i}}\right) * S\left(\frac{x}{\lambda z_{i}}\right)\right]\right\} * \delta\left(x-x_{i}\right) \\
& =\left[t_{i} h_{\alpha_{i}}(x) V(x)\right] * \delta\left(x-x_{i}\right) \\
& =t_{i} h_{\alpha_{i}}\left(x-x_{i}\right) V\left(x-x_{i}\right)
\end{aligned}
$$

where $\alpha_{i}=\frac{\pi}{\lambda z_{i}}=\frac{\pi}{\lambda\left(d+\Delta z_{i}\right)}$ and $V(x)=\left(j \lambda z_{i}\right)^{1 / 2} h_{-\alpha_{i}}(x) * S\left(\frac{x}{\lambda z_{i}}\right)$ where we used the Fourier transform property, $H_{\alpha_{i}}\left(\frac{x}{\lambda z_{i}}\right)=$ $\left(j \frac{\pi}{\alpha_{i}}\right)^{1 / 2} \exp \left(-j \frac{\left(2 \pi x /\left(\lambda z_{i}\right)\right)^{2}}{4 \alpha_{i}}\right)=\left(j \lambda z_{i}\right)^{1 / 2} h_{-\alpha_{i}}(x), \quad$ for $\quad$ quadratic 
phase functions given in [20]. The propagated field is the low pass filtered version of $h_{\alpha}(x)$ by the pixel function $s(x)$. The samples of this field,

$$
h_{L P_{D_{i}}}[n]=\left.h_{L P_{i}}(x)\right|_{x=n X},
$$

represent the discrete pixel values on the SLM. Each such sample is then multiplied by the sampled lenslet array phase distribution $L A[n]$, which is given by

$$
L A_{D}[n]=\left[p(x) \psi(x) * \sum_{r} \delta\left(x-r x_{0}\right)\right]_{x=n X} .
$$

The resulting discrete complex field is converted to an analog signal by convolving with the pixel function. Next, this analog complex field is propagated for a distance $g$ to find the field on the recording plane. Finally, we take the magnitude square of the field to simulate the intensity recording.

First let us look at the impulse response of the system for an impulse located at $\left(x_{i}, z_{i}\right)$. The impulse response of the capture system can be written as

$$
q_{i}(x)=\left\{\sum_{n} h_{L P_{D_{i}}}[n] L A_{D}[n] \delta(x-n X)\right\} * s(x) * h_{\beta}(x),
$$

where $h_{\beta}(x)=\exp \left(j \beta x^{2}\right), \beta=\frac{\pi}{\lambda g}$. The pixel function, $s(x)$, converts the discrete pattern into continuous field by zero-order hold interpolation. Since the systems are linear, we can interchange the order of the convolutions $s(x)$ and $h_{\beta}(x)$, given as

$$
q_{i}(x)=\left\{\sum_{n} h_{L P_{D_{i}}}[n] L A_{D}[n] h_{\beta}(x-n X)\right\} * s(x) .
$$

So, we first find the propagation result, where the details of the derivation are given in Appendix B. The final result of the impulse response can be written as (see Appendix B)

$$
q_{i}(x)=P_{i}\left(\frac{x}{\lambda g}\right) * s(x) *\left[\sum_{k} a_{k} H_{-\gamma_{k}}\left(\frac{x}{\lambda g}\right)\right] * \Psi(x) * \Upsilon_{i}(x)
$$

In Eq. (17), the last term

$$
\begin{aligned}
\Upsilon_{i}(x)= & \sum_{r} c(x)\left\{\left\{v\left(\frac{x}{\lambda g}\right) \exp \left[j 2 \beta\left(x_{i}-r x_{0}\right) x\right]\right\}\right. \\
& \left.* \delta\left[x-\left(1+\frac{g}{z_{i}}\right) r x_{0}+\frac{g}{z_{i}} x_{i}\right]\right\}
\end{aligned}
$$

where we obtain $v\left(\frac{x}{\lambda g}\right)=j \lambda z_{i} h_{\beta}\left(\sqrt{\frac{z_{i}}{g}} x\right) s\left(-\frac{z_{i}}{g} x\right)$ by using again the Fourier transform property, $H_{-\alpha_{i}}\left(\frac{x}{\lambda g}\right)=\left(j \frac{\pi}{\alpha_{i}}\right)^{1 / 2}$ $\exp \left(j \frac{(2 \pi x /(\lambda g))^{2}}{4 \alpha_{i}}\right)=\left(j \lambda z_{i}\right)^{1 / 2} h_{\beta}\left(\sqrt{\frac{z_{i}}{g}} x\right)$, for quadratic phase functions given in [20] and $c(x)=h_{\beta}\left(x-r x_{0}\right) t_{i} h_{\alpha_{i}}\left(x_{i}-r x_{0}\right)$, is a weighted impulse train that gives the perfect mapping (imaging) at locations $\left(1+\frac{g}{z_{i}}\right) r x_{0}-\frac{g}{z_{i}} x_{i}$ of the input point to multiple output points. This would be the imaging of a lenslet array consisting of perfect thin lenses. However, because of the low pass filtering caused by the pixel function at the input, this term gives blurred spots. The term $\Psi(x)$, which is a scaled impulse train due to sampling of the lenslets, causes multiple diffraction orders and it replicates the points at locations $\left(1+\frac{g}{z_{i}}\right) r x_{0}-\frac{g}{z_{i}} x_{i}$, where $\left(1+\frac{g}{z_{i}}\right) x_{0}$ is the elemental image separation and $-\frac{g}{z_{i}} x_{i}$ is the image point location. $\Psi(x)$ is given as

$$
\Psi(x)=x_{g} \sum_{n} \delta\left(x-n x_{g}\right),
$$

where $x_{g}=\frac{\lambda g}{X}$. Substituting $(1 / f-1 / d)^{-1}$ for $g$ we get $x_{g}=\left(\frac{1}{1-f / d}\right) x_{0}$, which is equal to $(1+g / d) x_{0}$. For sufficiently large $d$ and small object depth, the elemental image separation $\left(1+\frac{g}{z_{i}}\right) x_{0} \approx\left(1+\frac{g}{d}\right) x_{0}$. So, we get nearly the same separation periodicity for the elemental images and the higher diffraction orders of the elemental images. For those object points near to the distance $d$, we will have elemental images, which are imaged well. For other object points, which are further away from $d$, the separation of the higher diffraction orders will be slightly less than the elemental images separation. This might cause intermingled elemental images. In reality, for the central diffraction order, a human observer may not notice this effect. However, for higher orders this artifact might be noticeable. Furthermore, these far away points might be out of focus at the imaging plane because of the limited depth of field, caused by the term $P_{i}\left(\frac{x}{\lambda g}\right)$, which will be explained later. Thus, for a certain setup, it is possible to obtain good elemental images by satisfying the above constraints. Moreover, higher diffraction orders will have less intensity because of the rectangular pixels and the SLMs' finite size, as discussed previously in Subsection 2.B. So, these artifacts will not disturb the elemental images at the central diffraction order. The term $\sum_{k} a_{k} H_{-\gamma_{k}}\left(\frac{x}{\lambda g}\right)$ is introduced because of the multiple focal point property of the lenslets. In fact, this is another artifact term at the main image plane caused by the out-of-focus small images formed at multiple image planes related to the focal distances $f / k$ of higher-order lenslets due to quantization. $s(x)$ is the pixel function, which introduces an inherent apodization [18]. The first term in Eq. (17), $P_{i}\left(\frac{x}{\lambda g}\right)=P\left(\frac{x}{\lambda g}\right) * H_{\theta_{i}}\left(\frac{x}{\lambda g}\right)$, is the generalized pupil function, which takes defocussing due to different depths of the point sources at the input plane into account. The function $H_{\theta_{i}}\left(\frac{x}{\lambda g}\right)$ is responsible for the defocussing. $P\left(\frac{x}{\lambda g}\right)$ is the Fourier transform of the pupil function. This function is a limiting factor for the extent of $q_{i}(x)$. The constant $\theta_{i}$ is given as $\beta+\alpha_{i}=-\frac{\pi}{\lambda} \frac{\Delta z_{i}}{d\left(d+\Delta z_{i}\right)}$. Therefore, the overall response of the system to the point cloud is given by

$$
q(x)=\sum_{i} q_{i}(x)
$$

by adding the response of each source in the point cloud. Here we assume that each point is a source and, therefore, the field on it is independent of other source points. Finally, in order to obtain the elemental images, we simulate the intensity recording process by taking the magnitude square of the $q(x)$ as

$$
I(x)=|q(x)|^{2}=\left|\sum_{i} q_{i}(x)\right|^{2}
$$

To simplify $I(x)$, we can assume that the elemental images do not overlap and $P\left(\frac{x}{\lambda g}\right) * s(x)$ quickly diminishes with respect to $x$. We can further assume that intensities of the responses of each point of the point cloud can be added. In Eq. (20), the magnitude square removes the phase terms $j h_{\beta}\left(\sqrt{\frac{z_{i}}{g}} x\right) \exp \left[j \beta\left(x_{i}-r x_{0}\right) x\right]$ and $c(x)$ in $\Upsilon_{i}(x)$ and 


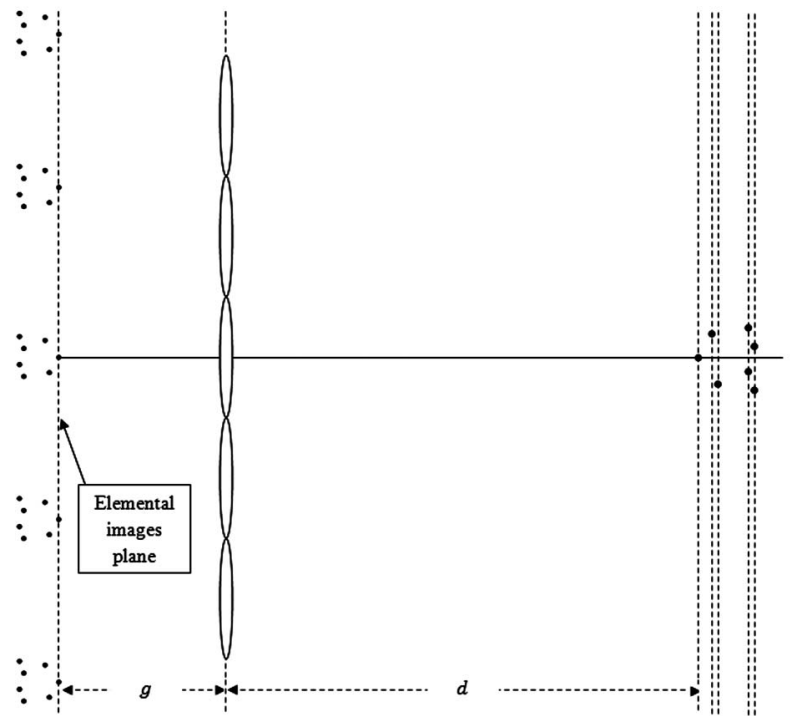

Fig. 5. Display setup.

$\left(\lambda z_{i}\right)^{2} s\left(-\frac{z_{i}}{g} x\right)$ remains as the only convolving term. Also, the impulse train $\Psi(x)$ introduces a constant weight, $x_{g}^{2}$, after the magnitude square operation. These impulses specify the locations of the imaged points. Thus, the impulse train indicated by $\Psi(x)$ and the impulse train in $\Upsilon_{i}(x)$ in Eq. (17) can be taken out of the magnitude square operation. The spot size of the imaged points are determined by convolution of the magnitude square of the function $\Gamma(x)=P_{i}\left(\frac{x}{\lambda g}\right) * s(x) *$ $\sum_{k} a_{k} H_{-\gamma_{k}}\left(\frac{x}{\lambda g}\right)$ and $\left(\lambda z_{i}\right)^{2} s\left(-\frac{z_{i}}{g} x\right)$. So, Eq. (20) can be approximated as

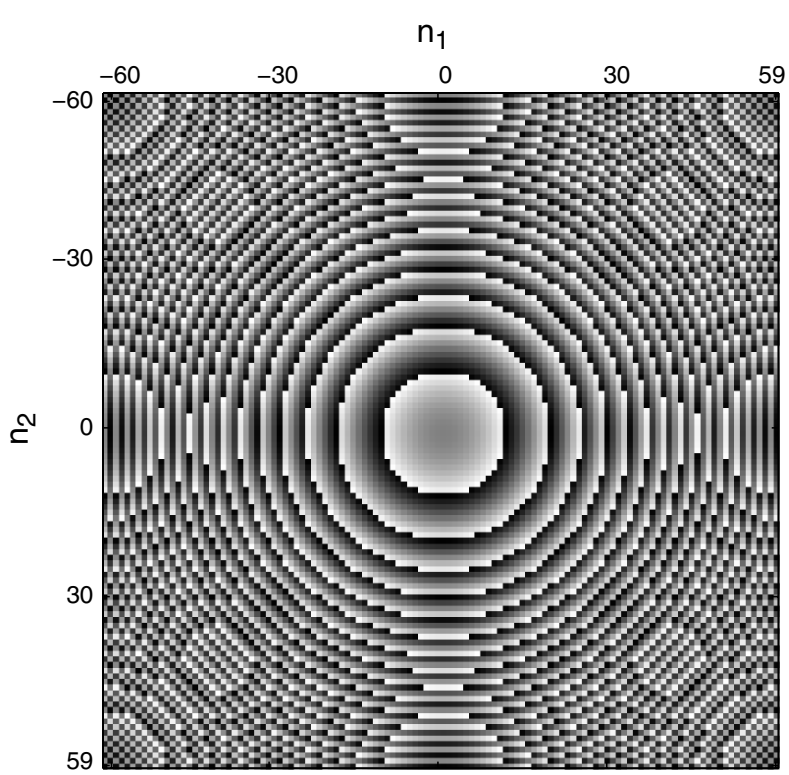

Fig. 6. Pixelated and quantized lens with $f=14.43 \mathrm{~mm}$. Sampling period is $8 \mu \mathrm{m}, \lambda=532 \mathrm{~nm}$, and array dimension is $120 \times 120$ pixels.

$$
\begin{aligned}
I(x) \approx & \sum_{i}\left\{|\Gamma(x)|^{2} *\left(\lambda z_{i}\right)^{2} s\left(-\frac{z_{i}}{g} x\right) * x_{g}^{2} \sum_{n} \delta\left(x-n x_{g}\right)\right. \\
& \left.* \sum_{r} \delta\left[x-\left(1+\frac{g}{z_{i}}\right) r x_{0}+\frac{g}{z_{i}} x_{i}\right]\right\} .
\end{aligned}
$$

To confirm this result we check the case where we use an analog lenslet array. If we have had an analog lenslet array, summation over $k$, the impulse train (indexed with $n$ ) due to sampling, the convolution with the pixel function $s(x)$ and the low-pass filtering with $\left(\lambda z_{i}\right)^{2} s\left(-\frac{z_{i}}{g} x\right)$ would be

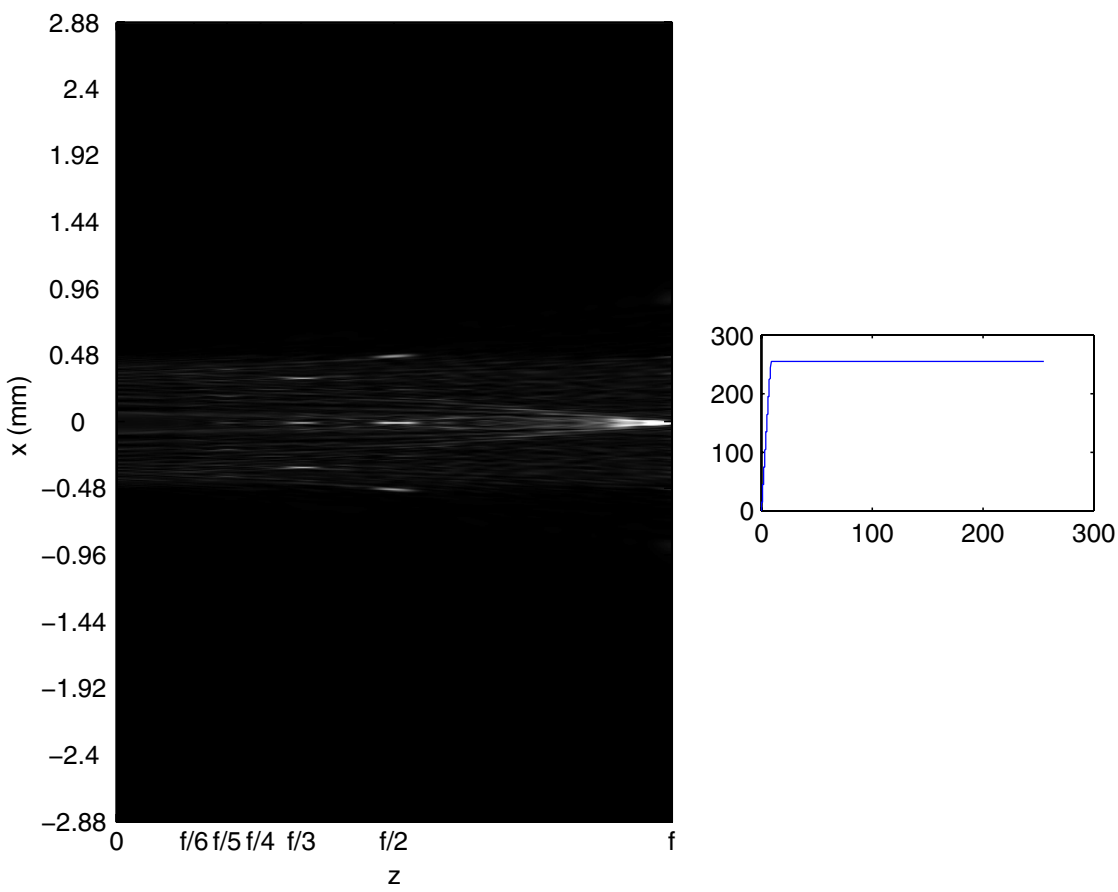

Fig. 7. (Color online) Magnitude square of the cross section of the field due to the pixelated and quantized lenslet, with $f=14.43 \mathrm{~mm}$, under plane wave illumination. The SLM is on the left. The bright areas indicate the multiple focal points and higher diffraction orders. (For visual purposes, we adjusted the brightness of the figure by stretching the contrast. The adjustment is given by the small graph. The horizontal axis represent the original gray values of the pixels, whereas the vertical axis is the modified gray value. We used a similar enhancement procedure also in Figs. $\underline{9}$ and $\underline{11}-\underline{14}$.) 


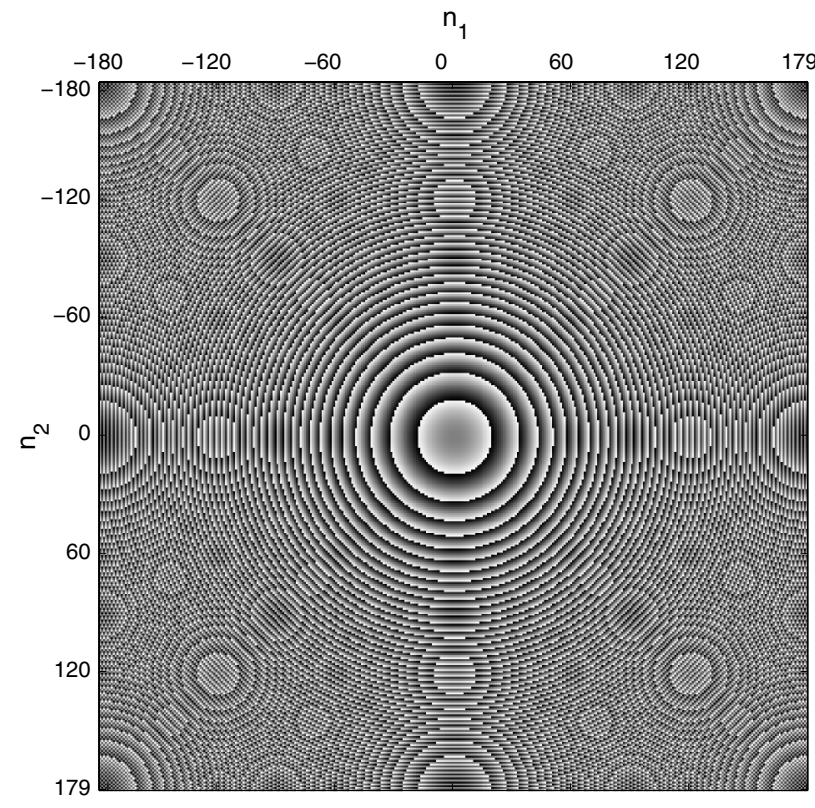

Fig. 8. Sampled lens with $f=43.3 \mathrm{~mm}$. Sampling period is $8 \mu \mathrm{m}$, $\lambda=532 \mathrm{~nm}$, and array dimension is $360 \times 360$ pixels.

dropped in Eq. (21). So, the result simplifies to the previous result given in $[\underline{4}, \underline{8}]$, which is given as

$$
I(x)=\sum_{i}\left\{\left|P_{i}\left(\frac{x}{\lambda g}\right)\right|^{2} * \sum_{r} \delta\left[x-\left(1+\frac{g}{z_{i}}\right) r x_{0}+\frac{g}{z_{i}} x_{i}\right]\right\} .
$$

\section{Display System}

The reconstruction process is similar to the capture process where the distances $g$ and $d$ are interchanged, that is, the distance between the input, in this case a $2 \mathrm{D}$ plane consisting of an array of elemental images, and the lenslet array is measured as $g$, while the distance between the reference plane on the reconstructed object/scene and the lenslet array is $d$. This is shown in Fig. 5. We can think that the light distribution $I(x)$ is input to this system. The input is only on a single plane consisting of a point cloud whereas the output consists of several planes. Each point in the point cloud will be reconstructed by carrying out a similar derivation as in Subsection 2.C. The object is perceived with a pseudoscopic 3D reconstruction, that is, the points nearer to the pickup lenses will be far away from the reconstruction lenses forming a depth reversed object.

\section{RESULTS}

\section{A. Computer Simulation Results}

As we mentioned in Subsection 2.B, we performed a series of computer simulations to show the multiple focuses and diffraction orders. First, we ran the simulations for a single lenslet. The lenslet profile is generated using Eq. (2). As described in Subsection 2.A, we determined $N$, the total number of samples along one dimension of the lenslet, by using the relation $f=N \frac{X^{2}}{\lambda}$, where $\lambda=532 \mathrm{~nm}$ and $X=8 \mu \mathrm{m}$. To obtain a single lenslet, we limit $n_{1}, n_{2}$ in Eq. (2) to be in the interval $\left[-\frac{N}{2}, \frac{N}{2}-1\right]$. For a lenslet with $f=14.4 \mathrm{~mm}, N$ is equal to 120 and for a lenslet with $f=43.3 \mathrm{~mm}, N$ is equal to 360 .

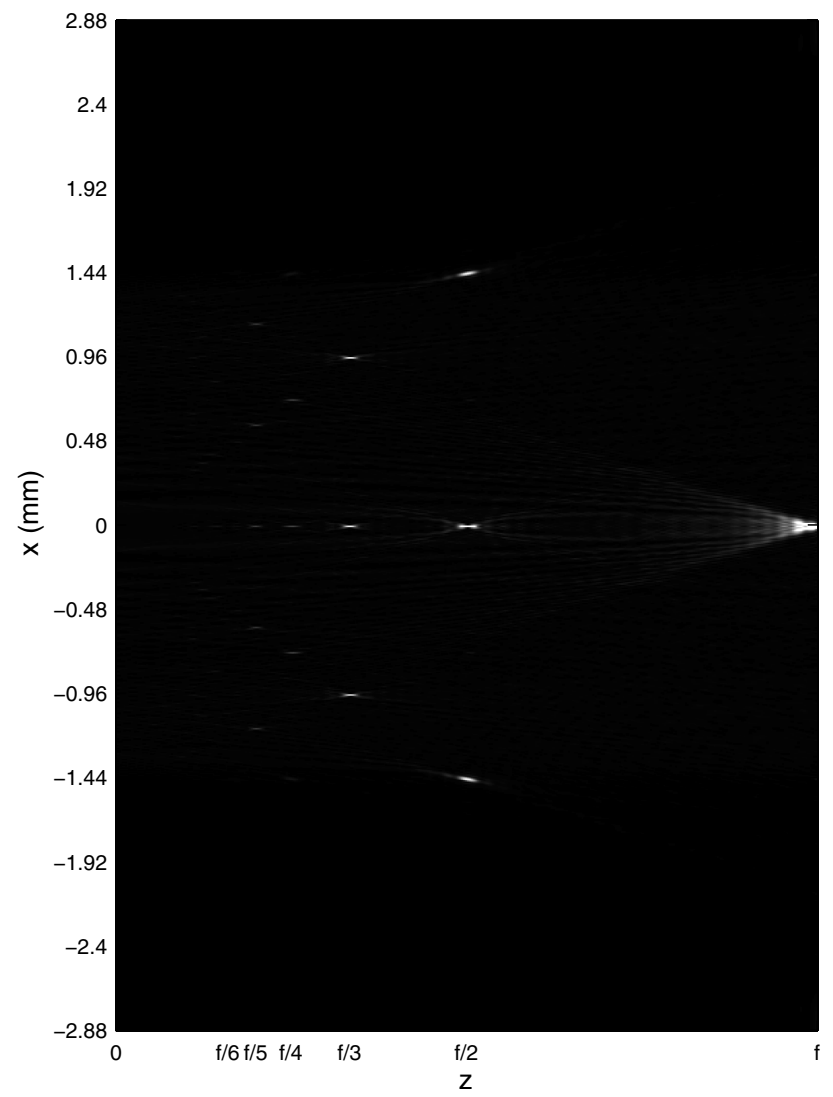

Fig. 9. Magnitude square of the cross section of the field due to the pixelated and quantized lenslet, with $f=43.3 \mathrm{~mm}$, under plane wave illumination. The bright areas indicate the multiple focal points and higher diffraction orders. The brightest area on the right is the fundamental focal point. (For visual purposes, we adjusted the brightness of the figure.)

We want to show the effect of quantization on the lenslet phase $(\bmod 2 \pi)$ by mapping this data linearly between 0 and 255. The results for these two single lenslets are shown in Figs. $\underline{6}$ and $\underline{8}$, respectively.

We assumed a plane wave as the incident light on the lenslets whose phase patterns are shown in Figs. $\underline{6}$ and $\underline{8}$. We performed wave propagation simulations using the Fresnel diffraction kernel. We computed the output of the propagation using convolution by discrete Fourier transform (DFT) method, which is given by

$$
d_{i}[\mathbf{n}]=\operatorname{IDFT}\left\{\operatorname{DFT}\{h[\mathbf{n}]\} H_{\chi_{i}}[\mathbf{k}]\right\},
$$

where $\mathbf{n}=\left[n_{1} n_{2}\right]^{T}$ are the discrete spatial domain variables and $\mathbf{k}=\left[k_{1} k_{2}\right]^{T}$ are the discrete spatial frequency domain variables, and $n_{1}, n_{2}, k_{1}, k_{2}$ are integers in the interval $[-360,359]$, and where $H_{\chi_{i}}[\mathbf{k}]$ is the 2D DFT of $h_{\chi_{i}}[\mathbf{n}]$ where $\chi_{i}$ is a parameter related to the propagation distance. IDFT denotes the inverse DFT. The multiplication inside 2D IDFT is a pointwise multiplication. DFT and IDFT operations are performed by two-dimensional fast Fourier transform (FFT) and inverse FFT algorithms, respectively. Convolution by the DFT method should be used carefully, as usual, to get linear convolution output using circular convolutions. We took the computation window size larger than the signal window in both directions and concatenated the signal window by zeros. Our computation window is 720 -sample wide in both 


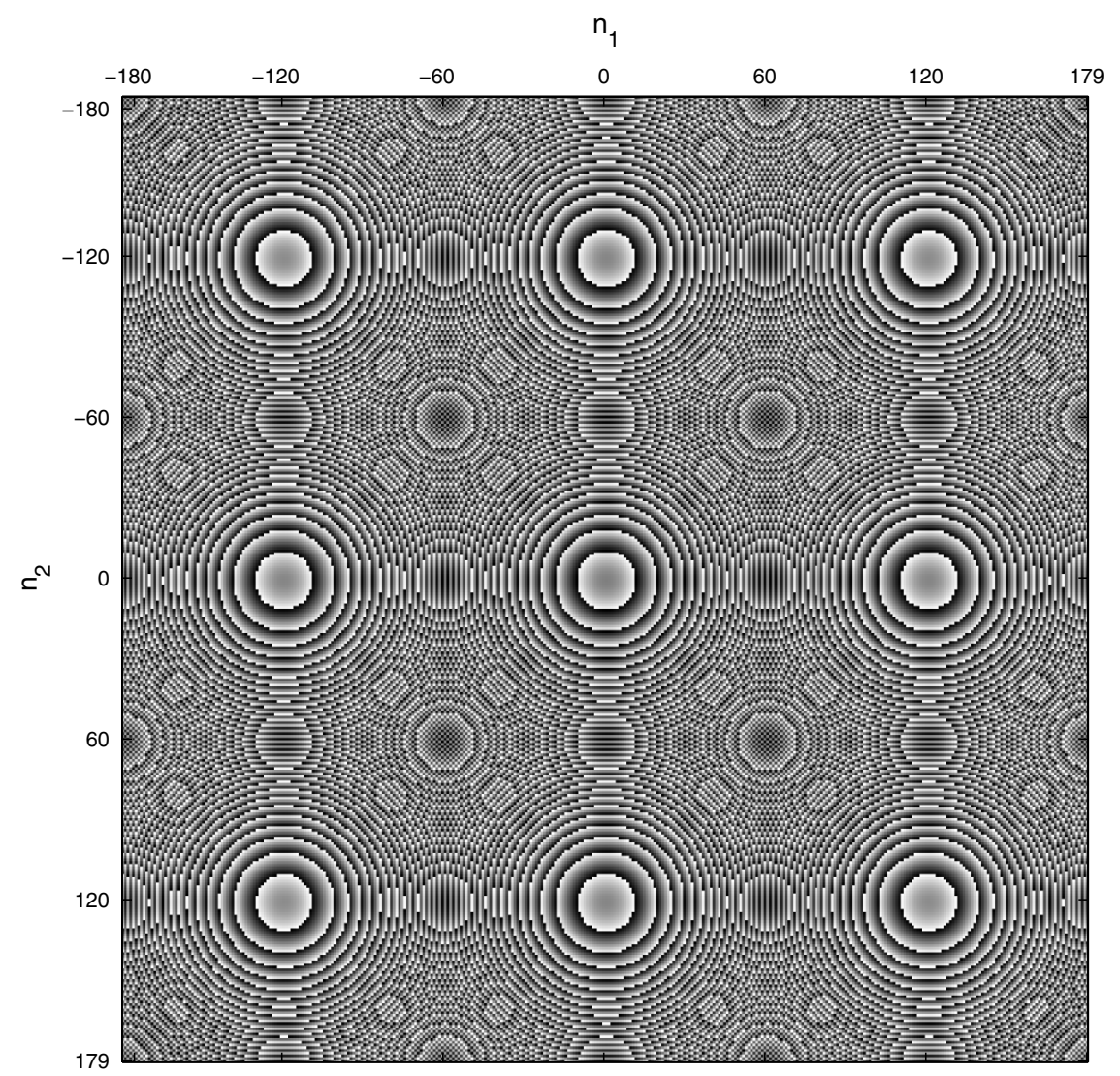

Fig. 10. Array of lenslets consisting of pixelated lenslets with $f=14.4 \mathrm{~mm}$. Total size is $360 \times 360$ pixels. Each lenslet in the array has the same properties defined as in Fig. 6 .

directions, which is six times the lenslet with $f=14.4 \mathrm{~mm}$ and two times the lenslet with $f=43.3 \mathrm{~mm}$.

The simulation calculates the field at certain distances until the fundamental focal point. So, we sampled the $z$ axis with equal separations of $z_{i}=i \frac{f}{L}$ in the interval $\left[0, \frac{(L-1)}{L} f\right]$, where $L=500$ is the total number of samples along the $z$ axis and each $\chi_{i}=\frac{\pi}{\lambda z_{i}}$. For each distance, $z_{i}$, a $2 \mathrm{D}$ diffraction pattern, $d_{i}[\mathbf{n}]$, is obtained. So, by combining all such $2 \mathrm{D}$ diffraction patterns, we obtain a 3D diffraction volume. We are interested in the locations of focal points. For display purposes, we take the $2 \mathrm{D}$ cross section of this $3 \mathrm{D}$ field, that is, we extracted the discrete values on the $n_{2}$ axis at $n_{1}=0$ at each $z$. The results of the propagation with the constraints explained above are shown in Fig. $\underline{7}$ and in Fig. 9 for lenslets with focal lengths 14.4 and $43.3 \mathrm{~mm}$, respectively. We converted the sample values in the $2 \mathrm{D}$ array to actual physical dimensions and labeled the axes in the figures, accordingly. The multiple focal points and diffraction orders are clearly seen in the $x z$ plane, as expected.

Now we proceed to simulate a lenslet array by limiting the lenslet array pattern in the interval $\left[-\frac{3 N}{2}, \frac{3 N}{2}-1\right]$ (three lenslets on each axis), as shown in Fig. 10. Again we simulated the propagation of the input lenslet array phase pattern illuminated by a plane wave. As in the previous simulations, we obtained the 2D $(x, z)$ cross section of the propagated field. The results of the free space propagation for this lenslet array is presented in Fig. 11. Each lenslet in the array has a focal length of $14.4 \mathrm{~mm}$. We used the same equation, Eq. (23), to calculate the diffraction field. This time, a periodic input pattern at the input yielded periodic diffraction orders of multiple focal points [20]. The figure shows that by adding more and more lenslets, we can observe the phenomenon as described in the analysis given in Subsection 2.B, where the theoretical results are presented by Eq. (10).

As mentioned in Subsection 2.B, we showed that the multiple image planes are associated with the multiple focal length phenomenon due to nonlinearity introduced by quantization. To show this effect, we used a $3 \times 3$ lenslet array, each lenslet having a focal length of $43.3 \mathrm{~mm}$. The window size of the lenslet array, $L A[\mathbf{n}]$, is $1080 \times 1080$ samples. The entire simulation window size is $1920 \times 1920$ samples. The lenslet array is centered in the computation array, $w_{L A}[\mathbf{n}]$, of size $1920 \times 1920$ samples with zeros padded around the lenslet array. We used a $2 \mathrm{D}$ input array, $t[\mathbf{n}]$, which is illuminated by a plane wave. The mask is letter "A", which is centered again in a computation array of size $1920 \times 1920$ samples. The background is black while the letter is white. We multiplied this mask with a normally distributed pseudorandom phase to simulate a diffuse object. To obtain the elemental images at the multiple image planes, we first computed the propagation result, $d_{1}[\mathbf{n}]$, from the input $t[\mathbf{n}]$ until the lenslet array plane, where the propagation distance is $4 f$. Then, we multiplied the propagated field with the pixelated and quantized lenslet array profile, $w_{L A}[\mathbf{n}]$. And then, we propagated the resulting pattern to two different distances $4 f / 3$ and $4 f / 7$ to obtain $d_{3}[\mathbf{n}]$ and $d_{4}[\mathbf{n}]$, respectively. The entire simulation can be summarized by 


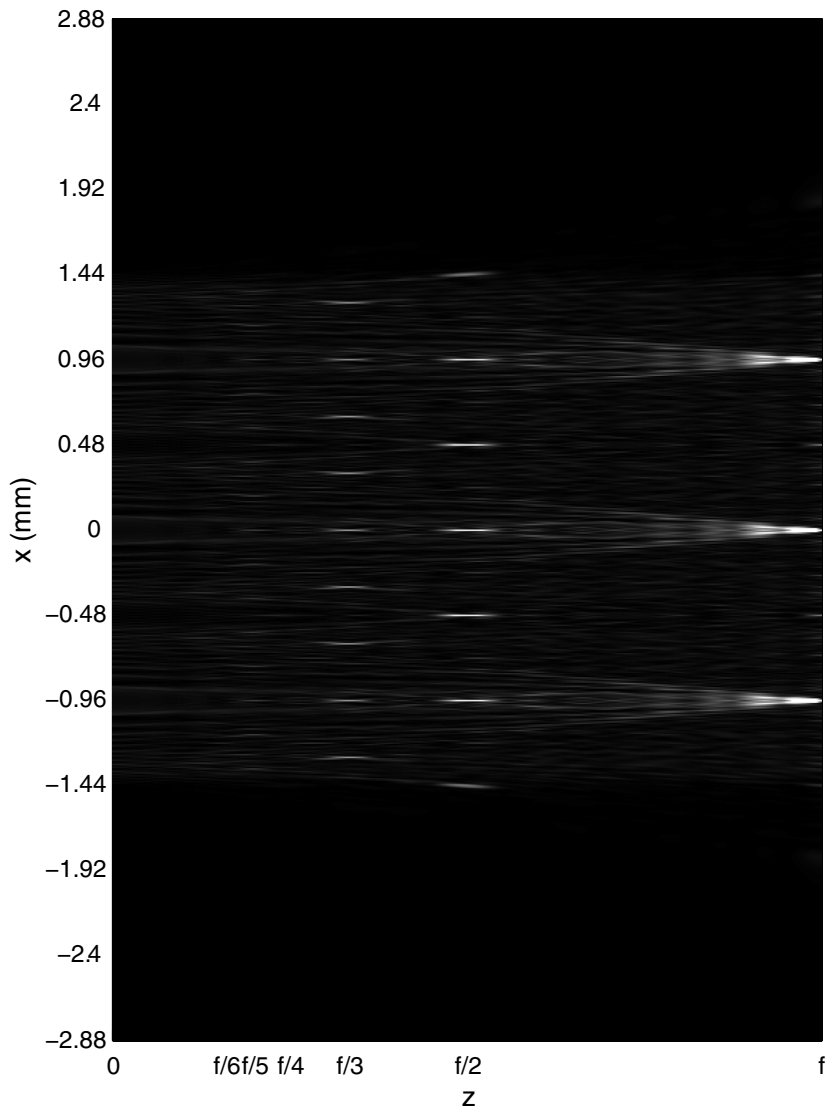

Fig. 11. Magnitude square of the cross section of the field due to the array of lenslets consisting of sampled lenslets, with $f=14.4 \mathrm{~mm}$, under plane wave illumination. Bright areas indicate the multiple focal points and higher diffraction orders. The brightest areas on the right are the fundamental focal points corresponding to each lenslet. (For visual purposes, we adjusted the brightness of the figure.)

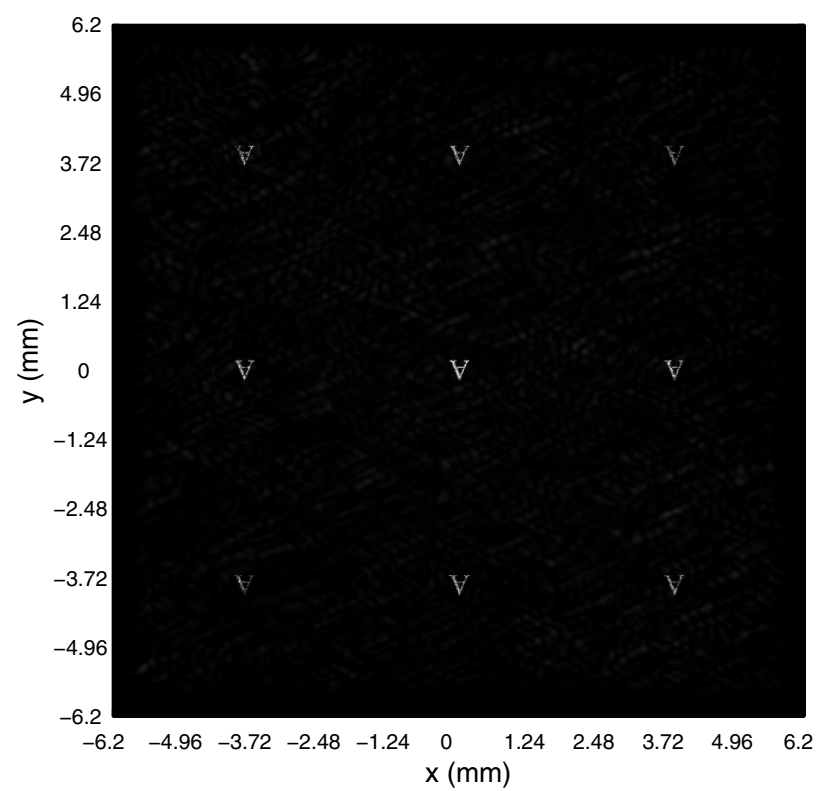

Fig. 12. Image of the absolute value of $d_{3}[\mathbf{n}]$. There are nine elemental images due to nine lenslets of the letter " $\mathrm{A}$ ". There is an artifact due to the out-of-focus images introduced by the multiple focal length properties of the lenslets. However, the visibility is still good. (For visual purposes, we adjusted the brightness of the figure by stretching the contrast using a procedure similar to the one described in Fig. 7 .)

$$
\begin{aligned}
& d_{1}[\mathbf{n}]=\operatorname{IDFT}\left\{\operatorname{DFT}\{t[\mathbf{n}]\} H_{\alpha}[\mathbf{k}]\right\}, \\
& d_{2}[\mathbf{n}]=d_{1}[\mathbf{n}] w_{L A}[\mathbf{n}], \\
& d_{3}[\mathbf{n}]=\operatorname{IDFT}\left\{\operatorname{DFT}\left\{d_{2}[\mathbf{n}]\right\} H_{\beta_{1}}[\mathbf{k}]\right\}, \\
& d_{4}[\mathbf{n}]=\operatorname{IDFT}\left\{\operatorname{DFT}\left\{d_{2}[\mathbf{n}]\right\} H_{\beta_{2}}[\mathbf{k}]\right\}
\end{aligned}
$$

where $\alpha=\frac{\pi}{\lambda 4 f}, \beta_{1}=\frac{\pi}{\lambda 4 f / 3}$, and $\beta_{2}=\frac{\pi}{\lambda 4 f / 7}$, and where $n_{1}, n_{2}, n_{3}$, $n_{4}$ are in the interval [-960,959]. In Fig. 12, the image obtained by taking the absolute value of $d_{3}[\mathbf{n}]$ is shown. As it can be seen from the figure that there are nine elemental images due to nine lenslets. The out-of-focus terms cause an artifact that is visible at the background of the figure at this distance. This artifact is caused by the multiple focal length properties of the lenslets and it is theoretically given by the term $\sum_{k} a_{k} H_{-\gamma_{k}}\left(\frac{x}{\lambda g}\right)$ in Eq. (21). At this image plane, which we call the main image plane, the images of "letter A" are brighter. Thus, their visibility is not disturbingly affected by the superimposed out-of-focus images. When we focus to the other distance, $4 f / 7$, we obtain Fig. 13 , which shows the absolute value of $d_{4}[\mathbf{n}]$. At this distance the "letter A" is again focused, as expected. On this plane, we observe 25 five images. Nine of them are shown in Fig. 9 by squares. These are due to the multiple focal point property of the lenslets. However, there are intermediate images between the images inside the squares. These are present due to the higher diffraction orders created by the pixelated structure of the lenslets. There are similar distortions at this imaging plane as in the main image plane. Since on this plane the images are smaller, thus they have small power, the out-of-focus fringes degrade the visibility significantly. So, a zoomed in version of the central elemental images and the intermediate image right to it are shown in Fig. 14.

\section{B. Optical Setup and Experimental Results}

We constructed a simplified integral imaging system and conducted experiments with this system to confirm the theoretical analysis and computer simulations given in Sections 2.C and 3.A, respectively. Since careful alignment of both parts (capture and display) are needed to match the elemental images captured by the CCD array to the LCD at the display setup to have a good reconstruction, we chose to construct our overall integral imaging system in one single stage, as shown in Fig. 15. The display part immediately follows the capture part in this experimental setup. A picture of the setup is shown in Fig. 16. Furthermore, in the optical setup, we used a green $10 \mathrm{~W} 4 \times \overline{5}$ LED array from Edipower, as the object instead of using a 3D object (Fig. 17), where we masked some LEDs to form the letter "C" shape. LEDs fit into a square of $7 \times 7 \mathrm{~mm}^{2}$ area. One reason for us to choose an LED array is the undiffracted light at the output of the display. Since the SLMs have a limited diffraction efficiency, the observable reconstruction will have a quite limited power. When we used a passive 3D object illuminated by an external source in the experiments, we were able to observe elemental images on the diffuser at the elemental images plane. However, since there is an undiffracted light at the background together with the elemental images and since the intensities of the elemental images are lower compared to the undiffracted light, the visibility was poor. So, it was difficult to observe the reconstruction with these elemental images from a passive (illuminated) object at the display part of the proposed setup. When we 


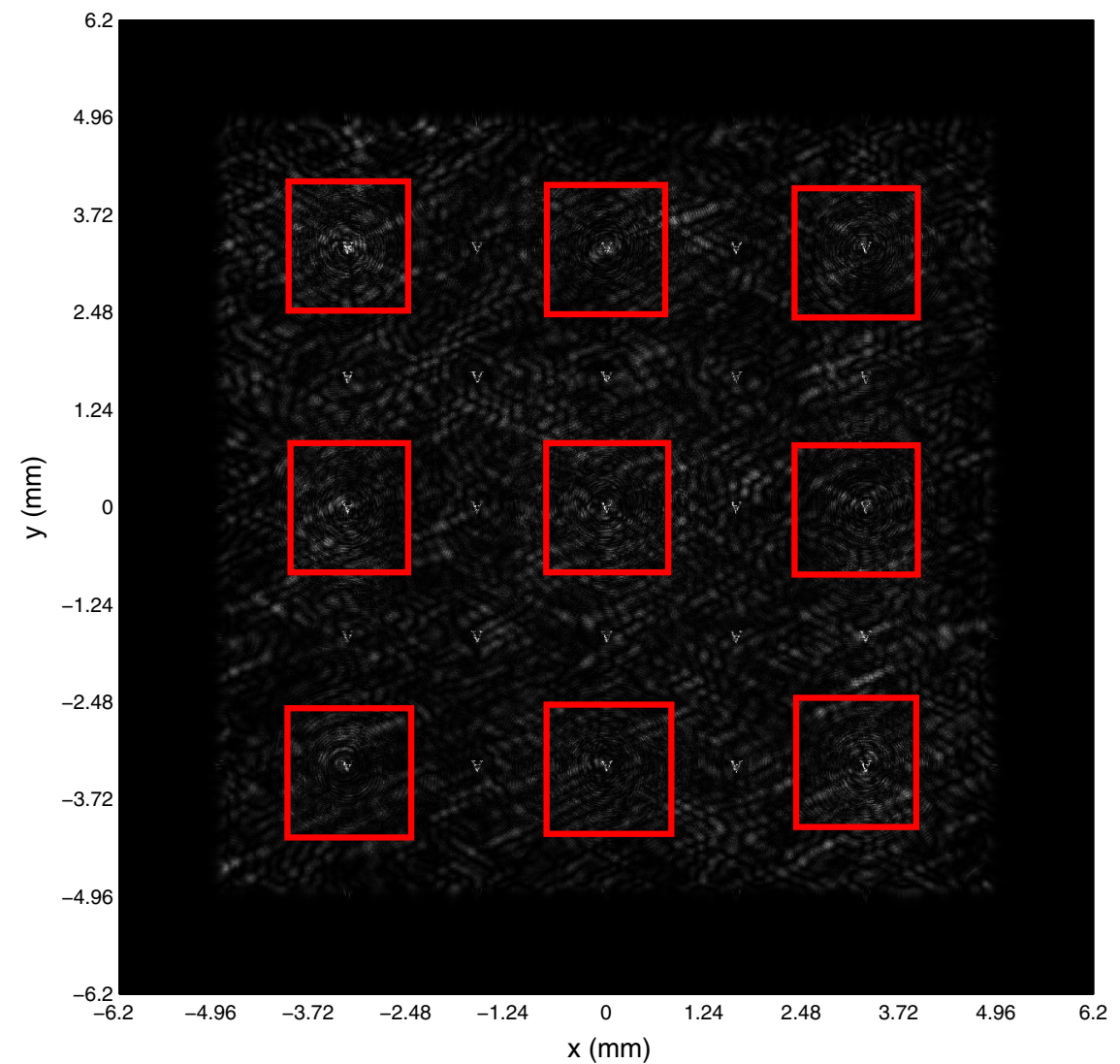

Fig. 13. (Color online) Image of the absolute value of $d_{4}[\mathbf{n}]$. The elemental images, which are depicted inside the rectangles, of the letter "A" are seen together with the higher diffraction orders between the elemental images. A zoomed-in version of the central elemental image is given in Fig. 14. We observe a similar artifact at the background. However, the visibility of elemental images are now degraded significantly due to this artifact. This is because of the smaller-size elemental images with less power. (For visual purposes, we adjusted the brightness of the figure.)

used the LED array we still observed the undiffracted light at the background. But this time, intensities of the focused images are much brighter. The other reason to use a singlecolor 2D LED array instead of a 3D object is the chromatic aberration introduced by the lenslets. Since, we calculated

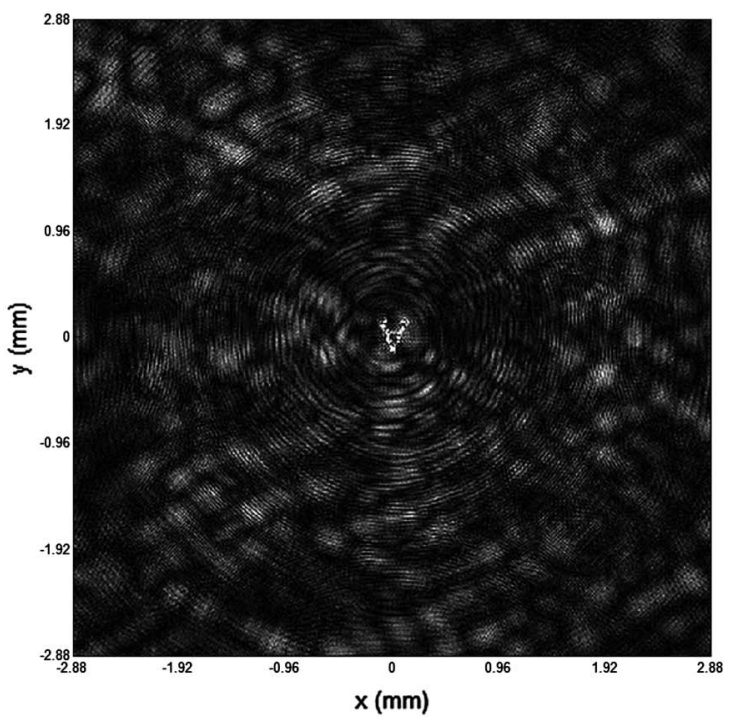

(a) the lenslets for a certain wavelength, $532 \mathrm{~nm}$, an object illuminated with a white light causes elemental images to have chromatic aberration. There are some proposed methods to compensate for chromatic aberration [22]. However, in our experiments we chose to use a self-luminous object with a

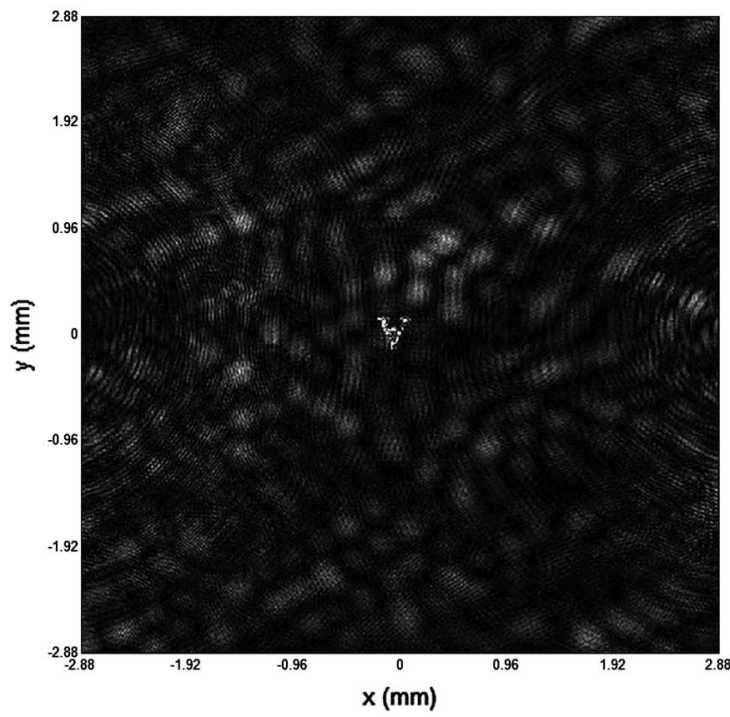

(b)

Fig. 14. (a) Zoomed-in elemental image corresponding to the central part of Fig. 13. (b) Zoomed-in elemental image corresponding to the image right to the central part of Fig. 13. (For visual purposes, we adjusted the brightness of the figure.) 


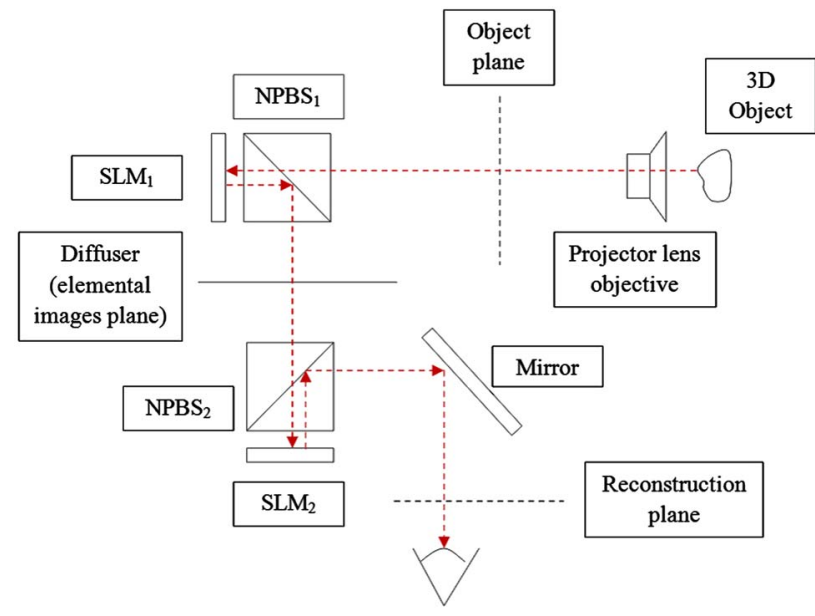

Fig. 15. (Color online) Experimental setup.

single color; our aim was to check the presented analysis. A single wavelength light source from a self-luminous LED array is easier to observe because the undiffracted light intensity will not dominate and there will be no chromatic aberrations. We used the lenslet array given in Fig. 1 on both SLMs in the capture and display part.

In the capture part, the object was imaged at the object plane with a projector lens, which is taken from an EPSON EMP TW-520 projector, to shrink the size of the real object and to collect and confine the light into a conical volume. The LED array is placed just behind the objective lens. The objective lens is adjusted such that the imaging distance from the objective to the object plane is $45 \mathrm{~mm}$. The image of the LEDs, shown in Fig. 18, covers a $4 \times 4 \mathrm{~mm}^{2}$ area on the object plane. The small sized real image of the object is then imaged by the lenslet array on $\mathrm{SLM}_{1}$ to the diffuser plane where we observed the elemental images shown in Fig. 19. The distance from the object plane to the surface of $\mathrm{SLM}_{1}$ is $188 \mathrm{~mm}$, and the distance from the SLM surface to the diffuser is $68 \mathrm{~mm}$. In theory, the lenslets should have exactly $43.3 \mathrm{~mm}$ focal length in free space propagation. However, in our system we use beam splitters in front of the SLMs, so, the actual focal length of the lenslets is shifted to approximately $55.3 \mathrm{~mm}$. So, we first try to find the elemental image plane (the plane where the elemental images seen the sharpest) and place the diffuser at this plane. And then, we measured the distance from the $\mathrm{SLM}_{1}$ surface to the diffuser and placed the second SLM accordingly. The elemental images fit into a rectangle of approximately $10 \mathrm{~mm} \times 150 \mathrm{~mm}$ area. One elemental image size is about $2 \mathrm{~mm} \times 2 \mathrm{~mm}$. The visibility of the elemental image set was good. As discussed in Subsection 2.C, we were also able to see the higher diffraction orders caused by the pixelated structure of the SLM. These orders are intermingled because of the difference between the elemental image separation period and the higher diffraction order separation period. Because of the finite SLM size, the intensities of the first diffraction orders are weaker than the central order, but stronger compared to the other higher orders. We masked higher diffraction orders even if they do not strongly affect the display part.

The display part starts from the diffuser. Since the elemental images were imaged directly on the diffuser, we did not need any other display device like in the conventional systems. So, we were able to observe the reconstructed image, which is shown in Fig. 20, by directly looking at the second lenslet array on $\mathrm{SLM}_{2}$. We put another diffuser at the reconstruction distance. The distance of this diffuser to the elemental image plane is the same as the distance from the object plane to the elemental images plane. The reason for this second diffuser is to show that the reconstructed image is real. In fact, we were able to see the reconstruction well with bare eye without any diffuser. The quality of the reconstruction was quite high since we did not use a pixelated device (i.e., LCD) to display the elemental images. However, the resulting intensity is lowered by the beam splitters. Each beam splitter in the system lowers the input light intensity by at least half

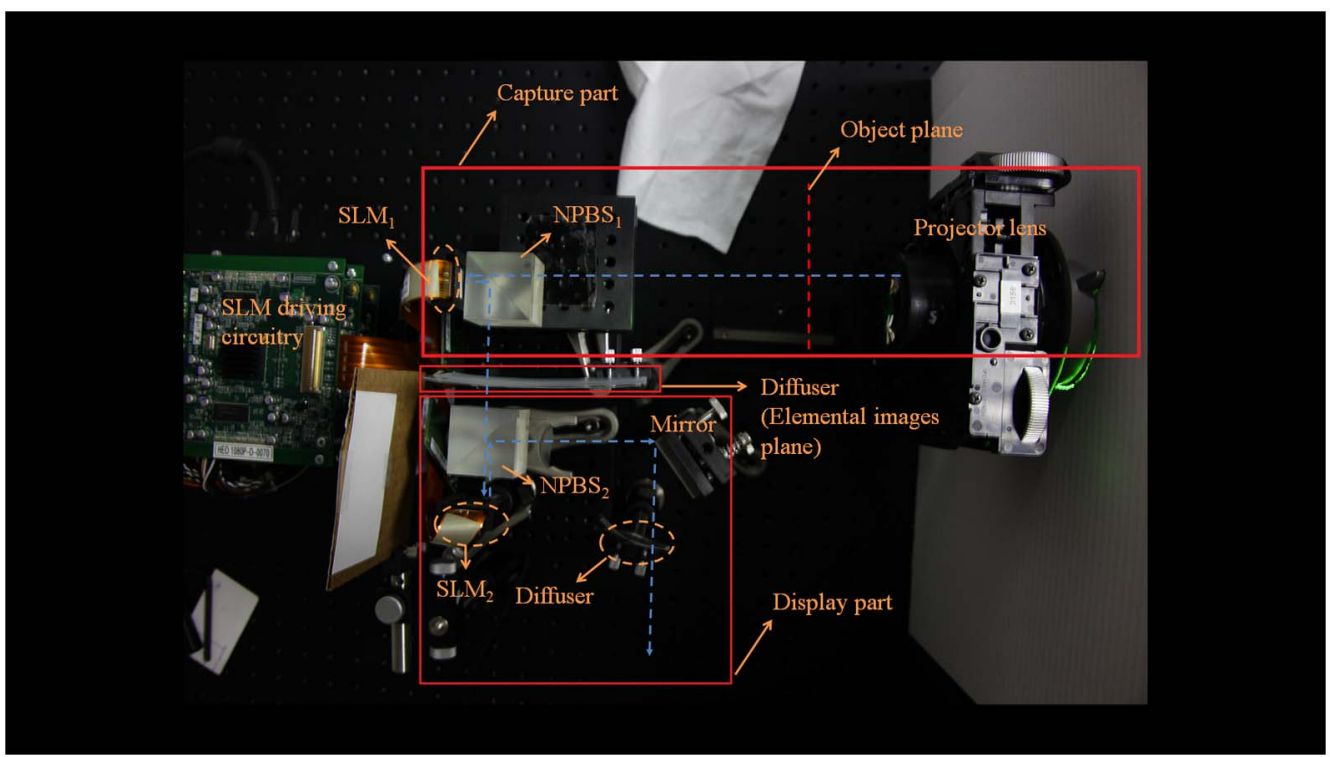

Fig. 16. (Color online) Top view of the optical setup: upper rectangle shows the capture part and lower square shows the display part. In between, a small rectangle shows the diffuser, which acts as a capture and display device, on the elemental images plane. The object is behind the white cardboard on the right before the projector lens. The cardboard prevents the light from the LED array to spread everywhere. The vertical dashed line after the projector lens shows the object plane. Dashed lines with the arrows show the optical path. The small diffuser after the mirror is used to show that the image at the calculated reconstruction distance is real. 


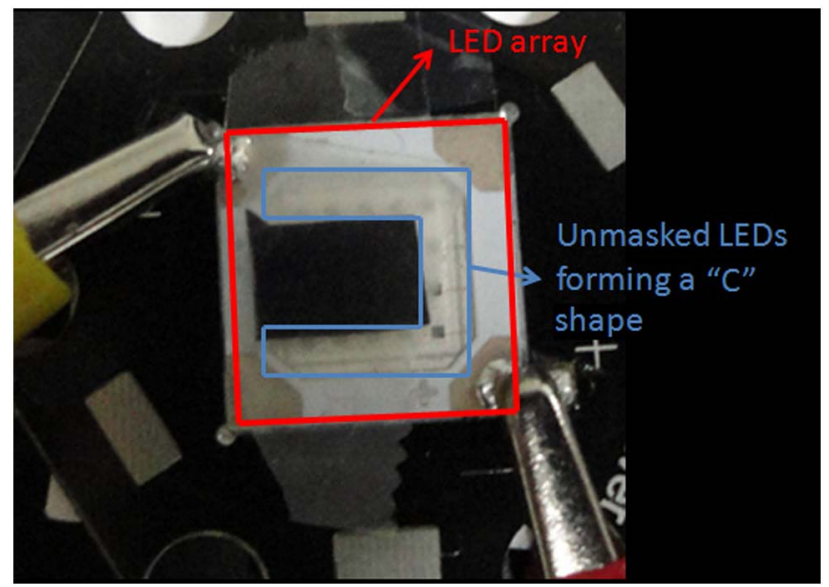

Fig. 17. (Color online) LED array that we used as the object. We put a black mask over the inner LEDs to form a (mirror image) "C" shaped object.

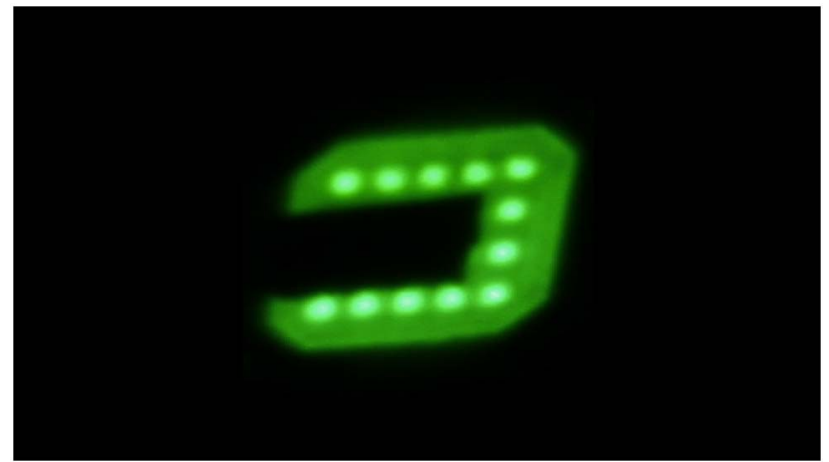

Fig. 18. (Color online) An image of the LED array on the object plane: the object is first imaged onto this plane by a projector lens to control both the depth and the size of the object.

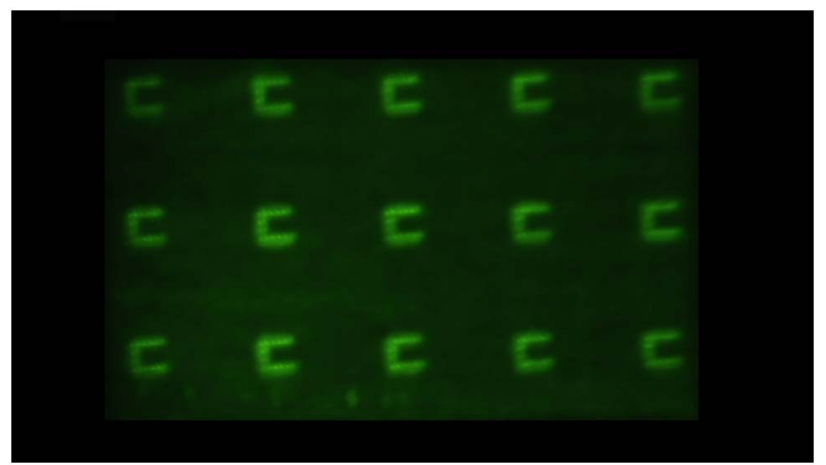

Fig. 19. (Color online) Optically captured elemental images.

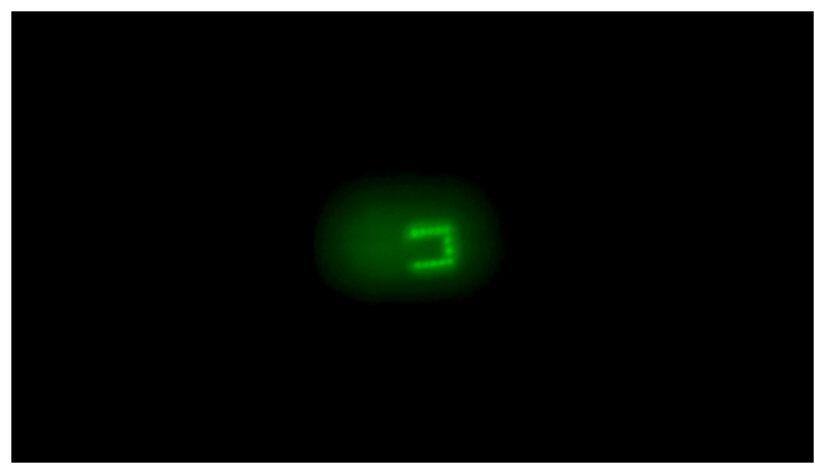

Fig. 20. (Color online) Optical reconstruction. and the light travels through each beam splitter twice. However, the light intensity at the output is acceptable. This experiment showed us that we can use phase-only SLMs with lenslet array phase pattern written on them to replace analog lenslet arrays in integral imaging systems. Such a system can easily be integrated into current digital projection systems.

\section{CONCLUSION}

We have reviewed the analysis of a pixelated and quantized lenslet. Such a lenslet causes multiple focal points and higher diffraction orders of these focal points at each focal distance. Multiple focal points are caused by the quantization, whereas the higher diffraction orders are caused by the pixelated structure.

When we look at the case of an array of such lenslets, we observe that the multiple focal points and higher diffraction orders from each such lenslet in the array come on top of each other if the period of the lenslet is chosen to be equal to the lenslet size, and the lenslets are generated such that they cover the entire normalized frequency range.

It is easy to generate numerically, any type of lenslet array by changing the physical parameters, like focal length, size, and even the phase distribution. So, certain drawbacks, like limited depth of focus, of a regular lenslet can be overcome. To realize these synthetic lenslet arrays physically, we need electronically controllable devices. One easy way to obtain such lenslet arrays is to write numerically generated Fresnel lenslet array patterns on a phase-only LCoS spatial light modulator. As a consequence, we obtain pixelated and quantized lenslet arrays.

Such a lenslet array can be used in an integral imaging system to replace the analog lenslet array both in the capture and display parts. We analyzed the capture part of such an integral imaging setup. When this lenslet array is used to image a 3D point cloud (in the capture part of an integral imaging system) we obtain an elemental image set at the main image plane. There are other image planes present due to multiple focal length property of pixelated and quantized lenslets. These higher-order images introduce additional artifact terms on the elemental images in addition to the out-of-focus term due to the limited depth of focus of the lenslets.

We performed wave propagation simulations for pixelated and quantized lenslets for two different focal lengths by using Fresnel diffraction kernel to support the theoretical analysis. In the simulations, we first looked at the case where a single lenslet is illuminated by a plane wave. We plotted the $2 \mathrm{D}$ cross section of the propagated field. For both lenslets we showed the multiple focal points together with higher diffraction orders. Then we looked at the case where we used small focal length lenslets to create an array with lenslet period equal to the lenslet size. We observed via simulations, multiple focal points and higher diffraction orders as indicated by the theoretical analysis. Finally, we used the large focal length lenslet to create another lenslet array, again with lenslet period equal to the lenslet size. We used this new lenslet array to image a diffuse mask. We obtained images of the mask at two different image planes. On the main image plane, the image is somewhat deteriorated. The artifact is caused by the multiple focal length property of the lenslets. Naturally, the shape and other optical properties of the mask directly affect the noisy looking background artifact. The diffusing nature of the mask results 
in a noise term that is well distributed over the image. We observed in our simulations that this noise term did not significantly degrade the visibility of the elemental images at this imaging plane. When we imaged the mask at a lower image plane, we observed the elemental images together with higher-order images in between them. The unwanted noisy looking background was still there, as expected, and the degradation in visibility was more significant in this plane. The reason for the increased degradation is the smaller size and lower intensity of the elemental images at this lower image plane.

To compare the theoretical and optical results, we constructed an integral imaging setup. In order to eliminate alignment problems between the capture and the display setups, we constructed the integral imaging setup such that the display part immediately follows the capture part and shares the elemental image plane. At the transition region (elemental image location), a diffuser was used instead of both capture (CCD) and display (LCD) devices. The crucial analog element of the system, that is, the lenslet array, was constructed as a phase pattern written on a phase-only LCoS SLM in both parts of the system. Since the SLMs are reflective type, we used beam splitters to split the incident wave and the reflected wave. However, these beam splitters changed the theoretical focal length of the lenslets. Thus, we modified the imaging distances accordingly. We first used a passive 3D object illuminated by an external source. However, because of the limited diffraction efficiency of the SLM device, there was an undiffracted light at the background together with the elemental images. Furthermore, the intensities of the elemental images were lower compared to this undiffracted light. So, this made it difficult to observe the reconstruction at the display part. Moreover, the lenslet array caused chromatic aberration since it is designed to work for a single wavelength. So we used a $2 \mathrm{D}$ green LED array, which is masked to form a letter, as the object. The system successfully worked to display this self-luminous object. The undiffracted background light was not disturbing when this self-luminous object was used. Experimental results show that the proposed system is feasible. Within the physical limitations of the system, we achieved good optical reconstruction. We have achieved an integral imaging system with a full digital capture and display lenslet arrays. Most of the integral imaging systems require a special type of lenslets (i.e., phase apodized) to improve the quality of the integral imaging system. These are generally hard and costly to manufacture. With our proposed system, it is possible to construct cost-efficient and simple integral imaging systems.

\section{APPENDIX A}

The convolution of the function inside the curly brackets and $h_{\chi}(x)$ in Eq. (9) can be written as

$$
\int\left[p(\eta) \psi(\eta) * \sum_{r} \delta\left(\eta-r x_{0}\right)\right] \sum_{n} \delta(\eta-n X) h_{\chi}(x-\eta) \mathrm{d} \eta .
$$

The above equation can be rewritten as

$$
\sum_{r} \int p\left(\eta-r x_{0}\right) \psi\left(\eta-r x_{0}\right) \sum_{n} \delta(\eta-n X) h_{\chi}(x-\eta) \mathrm{d} \eta,
$$

and by a change of variables as $\eta=\sigma+r x_{0}$ we get

$$
\begin{aligned}
\sum_{r} & \int p(\sigma) \psi(\sigma) \sum_{n} \delta\left(\sigma+r x_{0}-n X\right) h_{\chi}\left(x-\sigma-r x_{0}\right) \mathrm{d} \sigma \\
= & \sum_{r} \int p(\sigma) \psi(\sigma) \sum_{n} \delta\left(\sigma+r x_{0}-n X\right) h_{\chi}(x) \\
& \times \exp \left[-j 2 \chi x\left(\sigma+r x_{0}\right)\right] \exp \left[j \chi\left(\sigma+r x_{0}\right)^{2}\right] \mathrm{d} \sigma .
\end{aligned}
$$

Notice that $x_{0}$ in the impulse train is equal to $N X$, where $N$ is an integer. Therefore, $\sum_{n} \delta\left(\sigma+r x_{0}-n X\right)=\sum_{n} \delta(\sigma-n X)$ for each $r$. Further manipulations give

$$
\begin{aligned}
\sum_{r} \int & p(\sigma) \psi(\sigma) \sum_{n} \delta(\sigma-n X) h_{\chi}(x) \exp (-j 2 \chi x \sigma) \\
& \times \exp \left(-j 2 \chi x r x_{0}\right) h_{\chi}(\sigma) \exp \left(j 2 \chi r x_{0} \sigma\right) \exp \left(j \chi r^{2} x_{0}^{2}\right) \mathrm{d} \sigma \\
= & \sum_{k} a_{k} \int p(\sigma) h_{-\gamma_{k}}(\sigma) h_{\chi}(\sigma) \sum_{n} \delta(\sigma-n X) \\
& \times \sum_{r} h_{\chi}\left(x-r x_{0}\right) \exp \left(j 2 \chi r x_{0} \sigma\right) \exp \left(-j 2 \pi \frac{x}{\lambda z} \sigma\right) \mathrm{d} \sigma,
\end{aligned}
$$

where we used Eq. ( $\underline{5}$ ) for $\psi(\cdot)$. The summation over $k$ is due to the response of multiple lenses with focal lengths $f_{k}$. The above equation can be recognized as a Fourier transform, of a product of functions, from variable $\sigma$ to the spatial domain variable $\frac{x}{\lambda z}$. The Fourier transform can be written as

$$
\begin{aligned}
& \mathcal{F}_{\sigma \rightarrow \frac{x}{\lambda z}}\left\{\sum_{k} a_{k} p(\sigma) h_{-\gamma_{k}}(\sigma) h_{\chi}(\sigma) \sum_{n} \delta(\sigma-n X) \sum_{r} h_{\chi}\left(x-r x_{0}\right)\right. \\
& \left.\quad \times \exp \left(j 2 \chi r x_{0} \sigma\right)\right\},
\end{aligned}
$$

where we define the Fourier transform and the inverse Fourier transform as

$$
\begin{aligned}
& F(\nu)=\mathcal{F}_{x \rightarrow \nu}\{f(x)\}=\int_{-\infty}^{\infty} f(x) \exp (-j 2 \pi \nu x) \mathrm{d} x \\
& f(x)=\mathcal{F}_{\nu \rightarrow x}^{-1}\{F(\nu)\}=\int_{-\infty}^{\infty} F(\nu) \exp (j 2 \pi \nu x) \mathrm{d} \nu .
\end{aligned}
$$

Multiplications of the functions will result in convolutions after the Fourier transformation and we will get

$$
\begin{aligned}
\sum_{k} a_{k}\left\{P\left(\frac{x}{\lambda z}\right)\right. & * H_{-\gamma_{k}}\left(\frac{x}{\lambda z}\right) * H_{\chi}\left(\frac{x}{\lambda z}\right) * \frac{\lambda z}{X} \sum_{n} \delta\left(x-n \frac{\lambda z}{X}\right) \\
& \left.* \sum_{r} h_{\chi}\left(x-r x_{0}\right) \delta\left(x-r x_{0}\right)\right\}
\end{aligned}
$$

in the spatial domain. We are interested in those cases where $\chi=\gamma_{k}$, that is, $z=f_{k}$ in the above equation. Thus, we obtain

$$
\begin{aligned}
\sum_{k} a_{k}\{ & P\left(\frac{x}{\lambda f_{k}}\right) * H_{-\gamma_{k}}\left(\frac{x}{\lambda f_{k}}\right) * H_{\gamma_{k}}\left(\frac{x}{\lambda f_{k}}\right) \\
& \left.* \frac{\lambda f_{k}}{X} \sum_{n} \delta\left(x-n \frac{\lambda f_{k}}{X}\right) * \sum_{r} h_{\gamma_{k}}\left(x-r x_{0}\right) \delta\left(x-r x_{0}\right)\right\},
\end{aligned}
$$

where the convolution $H_{-\gamma_{k}}\left(\frac{x}{\lambda f_{k}}\right) * H_{\gamma_{k}}\left(\frac{x}{\lambda f_{k}}\right)=1$. The final result is thus, 


$$
\begin{aligned}
q(x)= & \sum_{k}\left\{\left[P\left(\frac{x}{\lambda f_{k}}\right) * \frac{x_{0}}{k} \sum_{n} \delta\left(x-\frac{n}{k} x_{0}\right)\right]\right. \\
& \left.*\left[\sum_{r} c_{k, r}(x) \delta\left(x-r x_{0}\right)\right]\right\} * s(x),
\end{aligned}
$$

where the constants are given as $\frac{\lambda f_{k}}{X}=\frac{N X}{k}=\frac{x_{0}}{k}$ and $c_{k, r}(x)=a_{k} h_{\gamma_{k}}\left(x-r x_{0}\right)$.

\section{APPENDIX B}

If we expand the quadratic phase function in Eq. (16), we will get

$$
\begin{aligned}
q_{i}(x)= & \left\{h_{\beta}(x) \sum_{n} h_{L P_{D_{i}}}[n] L A_{D}[n] h_{\beta}(n X) \exp (-j 2 \beta x n X)\right\} \\
& * s(x),
\end{aligned}
$$

where the summation is the so-called discrete time Fourier transform [24] of the function

$$
u[n]=h_{L P_{D_{i}}}[n] L A_{D}[n] h_{\beta}(n X),
$$

and $u_{c}(x)=h_{L P_{i}}(x) L A(x) h_{\beta}(x)$ is the continuous function of $u[n]$. Using the relation between the continuous time Fourier transform $F_{c}(\nu)$ of the continuous function $f_{c}(x)$ and the discrete time Fourier transform $F(\hat{\nu})$ of the discrete function $f[n]=f_{c}(n X)$, that is given by

$$
\left.F(\hat{\nu})\right|_{\hat{\nu}=\nu X}=F_{c}(\nu) * \frac{1}{X} \sum_{k} \delta\left(\nu-k \frac{1}{X}\right),
$$

where $\quad F(\hat{\nu})=\sum_{n} f[n] \exp (-j \hat{\nu} n) \quad$ and $\quad F_{c}(\nu)=\int_{-\infty}^{\infty} f_{c}(\eta)$ $\exp (-j 2 \pi \eta \nu) \mathrm{d} \eta$. We can rewrite $q_{i}(x)$ as

$$
q_{i}(x)=\left[h_{\beta}(x) U_{c}\left(\frac{x}{\lambda g}\right)\right] * x_{g} \sum_{n} \delta\left(x-n x_{g}\right) * s(x),
$$

where $x_{g}=\frac{\lambda g}{X}$. The scaled impulse train is due to sampling of the lenslets that causes multiple diffraction orders. Up to this point we can explain the effect of sampled lenslets at the output plane. However, we need to further analyze $\left[h_{\beta}(x) U_{c}\left(\frac{x}{\lambda g}\right)\right]$ to observe the effects of quantization. To do that, we first assume that the tails of the replicas of the continuous Fourier transform of the function are small so that they introduce negligible aliasing when they are summed. This is in fact true, because while the light travels from and through the physical optical elements, it does not spread too much in space. Thus, light cannot extend to very high angles. It is usually confined into a certain region. This can be seen as an inherent low-pass filtering of the optical elements. Furthermore, the pixelated physical optical elements will diffract light into higher orders while the modulated light propagates. So, each replica will spread to a limited region and will be separated from each other by a certain distance. These two properties will result in reducing the aliasing components. Using the Fourier transform relations given above in Eq. ( $\underline{\mathrm{B} 1})$, we expand $h_{\beta}(x) U_{c}\left(\frac{x}{\lambda g}\right)$ as

$$
\begin{aligned}
h_{\beta}(x) & U_{c}\left(\frac{x}{\lambda g}\right) \\
= & h_{\beta}(x) \int h_{L P_{i}}(\eta) L A(\eta) h_{\beta}(\eta) \exp \left(-j 2 \pi \frac{x}{\lambda g} \eta\right) \mathrm{d} \eta \\
= & h_{\beta}(x) \int h_{L P_{i}}(\eta)\left[p(\eta) \psi(\eta) * \sum_{r} \delta\left(\eta-r x_{0}\right)\right] \\
& \times h_{\beta}(\eta) \exp \left(-j 2 \pi \frac{x}{\lambda g} \eta\right) \mathrm{d} \eta \\
= & h_{\beta}(x) \int\left[t_{i} h_{\alpha_{i}}\left(\eta-x_{i}\right) V\left(\eta-x_{i}\right)\right] \\
& \times\left[\sum_{r} p\left(\eta-r x_{0}\right) \psi\left(\eta-r x_{0}\right)\right] h_{\beta}(\eta) \exp \left(-j 2 \pi \frac{x}{\lambda g} \eta\right) \mathrm{d} \eta \\
= & h_{\beta}(x) \int t_{i} h_{\alpha_{i}}(\eta) \exp \left(-j 2 \alpha_{i} \eta x_{i}\right) \exp \left(j \alpha_{i} x_{i}^{2}\right) V\left(\eta-x_{i}\right) \\
& \times\left[\sum_{r} p\left(\eta-r x_{0}\right) \psi\left(\eta-r x_{0}\right)\right] h_{\beta}(\eta) \exp \left(-j 2 \pi \frac{x}{\lambda g} \eta\right) \mathrm{d} \eta .
\end{aligned}
$$

We make a change of variables $\eta=\sigma+r x_{0}$ as in Appendix $\underline{\mathrm{A}}$ to get

$$
\begin{aligned}
h_{\beta}(x) U_{c}\left(\frac{x}{\lambda g}\right)= & \sum_{r} h_{\beta}(x) t_{i} \int_{\sigma} h_{\alpha_{i}}\left(\sigma+r x_{0}\right) \exp \left[-j 2 \alpha_{i}\left(\sigma+r x_{0}\right) x_{i}\right] \\
& \times \exp \left(j \alpha_{i} x_{i}^{2}\right) p(\sigma) \psi(\sigma)\left[h_{\beta}(\sigma) \exp \left(j 2 \beta \sigma r x_{0}\right)\right. \\
& \left.\times \exp \left(j \beta r^{2} x_{0}^{2}\right)\right] \exp \left(-j 2 \beta x r x_{0}\right) \\
& \times \exp \left(-j 2 \pi \frac{x}{\lambda g} \sigma\right) V\left(\sigma+r x_{0}-x_{i}\right) \mathrm{d} \sigma
\end{aligned}
$$

and then we expand some of the quadratic phase functions

$$
\begin{aligned}
h_{\beta}(x) U_{c}\left(\frac{x}{\lambda g}\right)= & \sum_{r} h_{\beta}(x) t_{i} \int h_{\alpha_{i}}(\sigma) \exp \left(j 2 \alpha_{i} \sigma r x_{0}\right) \exp \left(j \alpha_{i} r^{2} x_{0}^{2}\right) \\
& \times \exp \left(-j 2 \alpha_{i} \sigma x_{i}\right) \exp \left(-j 2 \alpha_{i} x_{i} r x_{0}\right) \\
& \times \exp \left(j \alpha_{i} x_{i}^{2}\right) p(\sigma) \psi(\sigma) h_{\beta}(\sigma) \exp \left(j 2 \beta \sigma r x_{0}\right) \\
& \times \exp \left(j \beta r^{2} x_{0}^{2}\right) \exp \left(-j 2 \beta x r x_{0}\right) \\
& \times \exp \left(-j 2 \pi \frac{x}{\lambda g} \sigma\right) V\left(\sigma+r x_{0}-x_{i}\right) \mathrm{d} \sigma
\end{aligned}
$$

Notice that the terms $h_{\beta}(x) \exp \left(-j 2 \beta x r x_{0}\right) \exp \left(j \beta r^{2} x_{0}^{2}\right)$ can be rearranged as $h_{\beta}\left(x-r x_{o}\right)$. And also the terms $\exp \left(j \alpha_{i} r^{2} x_{0}^{2}\right)$ $\exp \left(-j 2 \alpha_{i} x_{i} r x_{0}\right) \exp \left(j \alpha_{i} x_{i}^{2}\right)$ can be put into the compact form $h_{\alpha_{i}}\left(x_{i}-r x_{0}\right)$. Rearranging the remaining exponential terms and gathering the summation terms over the variable $r$, we finally get

$$
\begin{aligned}
h_{\beta}(x) U_{c}\left(\frac{x}{\lambda g}\right)= & \int h_{\theta_{i}}(\sigma) p(\sigma) \psi(\sigma)\left[\sum_{r} c(x) V\left(\sigma+r x_{0}-x_{i}\right)\right. \\
& \left.\times \exp \left[j 2 \sigma\left(\theta_{i} r x_{0}-\alpha_{i} x_{i}\right)\right]\right] \\
& \times \exp \left(-j 2 \pi \frac{x}{\lambda g} \sigma\right) \mathrm{d} \sigma,
\end{aligned}
$$

where $\theta_{i}=\beta+\alpha_{i}=-\frac{\pi}{\lambda} \frac{\Delta z_{i}}{d\left(d+\Delta z_{i}\right)}$ and $c(x)=h_{\beta}\left(x-r x_{0}\right) t_{i} h_{\alpha_{i}}$ $\left(x_{i}-r x_{0}\right)$. The above equation can be rewritten as 


$$
\begin{aligned}
& \mathcal{F}_{\sigma \rightarrow \frac{x}{\lambda g}}\{ {\left[h_{\theta_{i}}(\sigma) p(\sigma)\right]\left[\sum_{k} a_{k} h_{-\gamma_{k}}(\sigma)\right]\left[\sum_{r} c(x) V\left(\sigma+r x_{0}-x_{i}\right)\right.} \\
&\left.\left.\times \exp \left[j 2 \sigma\left(\theta_{i} r x_{0}-\alpha_{i} x_{i}\right)\right]\right]\right\} .
\end{aligned}
$$

Since the terms inside the right brackets are multiplied, the result of the Fourier transform will be convolution of corresponding Fourier transformed terms in the scaled spatial domain.

The Fourier transform of the last term is a weighted impulse train, which gives the perfect mapping (imaging) at locations $\left(1+\frac{g}{z_{i}}\right) r x_{0}+\frac{g}{z_{i}} x_{i}$ of the input point to multiple output points. This would be the imaging of a lenslet array consisting of perfect thin lenses. However, because of the low-pass filtering caused by the pixel function at the input, this last term gives blurred spots. The Fourier transform of the function inside the last right brackets is given as

$$
\begin{aligned}
\mathbf{\Upsilon}_{i}(x)= & \sum_{r} c(x)\left\{\left\{v\left(\frac{x}{\lambda g}\right) \exp \left[j 2 \beta\left(x_{i}-r x_{0}\right) x\right]\right\}\right. \\
& \left.* \delta\left[x-\left(1+\frac{g}{z_{i}}\right) r x_{0}+\frac{g}{z_{i}} x_{i}\right]\right\},
\end{aligned}
$$

where we obtain $v\left(\frac{x}{\lambda g}\right)=j \lambda z_{i} h_{\beta}\left(\sqrt{\frac{z_{i}}{g}} x\right) s\left(-\frac{z_{i}}{g} x\right)$ by using the Fourier transform property, $H_{-\alpha_{i}}\left(\frac{x}{\lambda g}\right)=\left(j \frac{\pi}{\alpha_{i}}\right)^{1 / 2} \exp \left(j \frac{(2 \pi x /(\lambda g))^{2}}{4 \alpha_{i}}\right)$ $=\left(j \lambda z_{i}\right)^{1 / 2} h_{\beta}\left(\sqrt{\frac{z_{i}}{g}} x\right)$, for quadratic phase functions given in [20]. The Fourier transform of the second term $\sum_{k} a_{k} H_{-\gamma_{k}}\left(\frac{x}{\lambda g}\right)$ is introduced because of the multiple focal point property of the lenslets. In fact, this is another artifact term at the main image plane caused by the smaller images formed at image planes related to other focal distances, $f / k$, of higherorder lenslets. The Fourier transform of the first term will be $P_{i}\left(\frac{x}{\lambda g}\right)=P\left(\frac{x}{\lambda g}\right) * H_{\theta_{i}}\left(\frac{x}{\lambda g}\right)$ is the generalized pupil function, which takes blurring due to different depths of the point sources at the input plane into account. The function $H_{\theta_{i}}\left(\frac{x}{\lambda g}\right)$ is responsible for the defocussing. $P\left(\frac{x}{\lambda g}\right)$ is the Fourier transform of the pupil function. This function is also a limiting factor for the extent of $q_{i}(x)$. Finally, arranging all terms, we can write $q_{i}(x)$ as

$$
\begin{aligned}
q_{i}(x)= & P_{i}\left(\frac{x}{\lambda g}\right) * s(x) *\left[\sum_{k} a_{k} H_{-\gamma_{k}}\left(\frac{x}{\lambda g}\right)\right] \\
& * x_{g} \sum_{n} \delta\left(x-n x_{g}\right) * \Upsilon_{i}(x) .
\end{aligned}
$$

\section{ACKNOWLEDGMENTS}

This work is supported by the European Commission within FP7 under grant 216105 with the acronym Real 3D. A. Özgür Yöntem thanks TÜBITTAK for the scholarship he received during doctoral studies.

\section{REFERENCES}

1. G. Lippmann, "La photographie intégrale," C.R. Hebd. Seances Acad. Sci. 146, 446-451 (1908).
2. H. E. Ives, "Optical properties of a Lippman lenticulated sheet," J. Opt. Soc. Am. 21, 171-176 (1931).

3. H. Hoshino, H. A. Jun, F. Okano, and I. Yuyama, "Real-time pickup method for a three-dimensional image based on the integral photography," Appl. Opt. 36, 1598-1603 (1997).

4. M. Martínez-Corral, B. Javidi, R. Martínez-Cuenca, and G. Saavedra, "Integral imaging with improved depth of field by use of amplitude-modulated microlens arrays," Appl. Opt. 43, 5806-5813 (2004).

5. Y. Frauel, A. Castro, and B. Javidi, "Integral imaging with large depth of field using an asymmetric phase mask," Opt. Express 15, 10266-10273 (2007).

6. S.-W. Min, S. Jung, H. Choi, Y. Kim, J.-H. Park, and B. Lee, "Wide-viewing-angle integral three-dimensional imaging system by curving a screen and a lens array," Appl. Opt. 44, 546-552 (2005).

7. D.-H. Shin, B.-G. Lee, J. Hyun, D.-C. Hwang, and E.-S. Kim, "Curved projection integral imaging using an additional largeaperture convex lens for viewing angle improvement," ETRI J. 31, 105-110 (2009).

8. M. Martinez-Corral, W. D. Furlan, and B. Javidi, "Analysis of 3-D integral imaging displays using the Wigner distribution," J. Disp. Technol. 2, 180-185 (2006).

9. H. Hoshino, J. Arai, F. Okano, and I. Yuyama, "Gradient-index lens-array method based on real-time integral photography for three-dimensional images," Appl. Opt. 37, 2034-2045 (1998).

10. S. H. Eng, Z. Wang, and K. Alameh, "Design and optimization of programmable lens array for adaptive optics," Proc. SPIE 6414, 64140K (2007).

11. L. Zhao, N. Bai, X. Li, L. S. Ong, Z. P. Fang, and A. K. Asundi, "Efficient implementation of a spatial light modulator as a diffractive optical microlens array in a digital Shack-Hartmann wavefront sensor," Appl. Opt. 45, 90-94 (2006).

12. J. Campos, E. Carcole, and S. Bosch, "Diffraction theory of Fresnel lenses encoded in low resolution devices," Appl. Opt. 33, 162-174 (1994).

13. M. K. Giles, "Applications of programmable spatial light modulators," presented at the International Conference on Lasers '96, Society of Optical and Quantum Electronics, 2-6 Dec. 1996.

14. X. Wang, H. Dai, K. X. Y. Liu, and J. Liu, "Characteristics of lcos phase only spatial light modulator and its applications," Opt. Commun. 238, 269-276 (2004)

15. R. A. Lilly, J. A. Davis, D. M. Cottrell, and S. W. Connely, "Multiplexed phase-encoded lenses written on spatial light modulators," Opt. Lett. 14, 420-422 (1989).

16. T. R. Hedman, D. M. Cottrell, J. A. Davis, and R. A. Lilly, "Multiple imaging phase-encoded optical elements written as programmable spatial light modulators," Appl. Opt. 29, 2505-2509 (1990).

17. J.-S. Jang and B. Javidi, "Three-dimensional integral imaging with electronically synthesized lenslet arrays," Opt. Lett. 27, 1767-1769 (2002).

18. A. Marquez, J. C. Escalera, J. A. Davis, C. Iemmi, M. J. Yzuel, J. Campos, and S. Ledesma, "Inherent apodization of lenses encoded on liquid-crystal spatial light modulators," Appl. Opt. 39, 6034-6039 (2000).

19. Y. Jeong, S. Jung, J.-H. Park, and B. Lee, "Reflection-type integral imaging scheme for displaying three-dimensional images," Opt. Lett. 27, 704-706 (2002).

20. L. Onural, "Some mathematical properties of the uniformly sampled quadratic phase function and associated issues in digital Fresnel diffraction simulation," Opt. Eng. 43, 2557-2563 (2004).

21. J. Jahns and S. J. Walker, "Two-dimensional array of diffractive microlenses fabricated by thin film deposition," Appl. Opt. 29, 931-936 (1990).

22. J. Oton, M. S. Millan, and E. Perez-Cabre, "Chromatic compensation of programmable Fresnel lenses," Opt. Express 14, 6226-6242 (2006).

23. L. Rayleigh, "On copying diffraction-gratings, and on some phenomena connected therewith," Philos. Mag. 11, 196-205 (1881)

24. A. V. Oppenheim, A. S. Willsky, and S. H. Nawab, Signals and Systems Second Edition (Prentice-Hall, 1997). 